\title{
Review Article \\ Study on the Similarity Laws for Local Damage Effects in a Concrete Target under the Impact of Projectiles
}

\author{
Mingyang Wang, ${ }^{1,2}$ Zheng Liu, $^{1,3}$ Yanyu Qiu, ${ }^{1,2}$ and Cuncheng Shi ${ }^{1,4}$ \\ ${ }^{1}$ State Key Laboratory for Disaster Prevention \& Mitigation of Explosion \& Impact, PLA University of Science and Technology, \\ Nanjing 210007, China \\ ${ }^{2}$ School of Mechanical Engineering, Nanjing University of Science and Technology, Nanjing 210094, China \\ ${ }^{3}$ Engineering Research and Designing Institute of Xinjiang Military Area Command, Wulumuqi 830042, China \\ ${ }^{4}$ The Fifth Department, 145 Erqi Road, Wuhan 430012, China
}

Correspondence should be addressed to Zheng Liu; 422297513@qq.com

Received 30 April 2015; Revised 15 August 2015; Accepted 16 August 2015

Academic Editor: Vadim V. Silberschmidt

Copyright (C) 2015 Mingyang Wang et al. This is an open access article distributed under the Creative Commons Attribution License, which permits unrestricted use, distribution, and reproduction in any medium, provided the original work is properly cited.

\begin{abstract}
The local destruction and deformation characteristics of a concrete target impacted by a rigid projectile were analyzed, and the similarity laws for local damage effects in the concrete target were studied utilizing the rigid-plastic, internal friction, and modified hydrodynamic models. For a thin target, the impact factor is the only factor controlling the low-velocity impact process. For a thick target impacted by a projectile at intermediate velocity, internal friction is the main factor contributing to the energy dissipation. The impact factor, the toughness factor, and the dynamic factor together determine the penetration process. However, for a thick target impacted at high velocity, the impact factor and hardness factor together determine the penetration process. The penetration depth shows a $2 / 3$ power relationship with impact velocity. For thick targets, similarity laws change along with impact velocity. The radii ratio between the projectile and penetration tunnel is proportional to the projectile's diameter for intermediate velocity impact and only shows a relationship with the impact velocity for high velocity penetration.
\end{abstract}

\section{Introduction}

As the main construction material, concrete is widely used in civil and military engineering. Studies on destruction effects in concrete structures have drawn widespread attentions, especially after World War II. The investigation of damage effects on concrete structures under impact has become a major research field in the weapon manufacturing industry and protective engineering and continues to receive prevalent attention from research groups all over the world.

For a finite thickness target impacted by a rigid projectile, overall and local effects appear as the striking velocity increases. Scabbing, perforation, and penetration are the main types of local effects [1]. The corresponding methods of calculating the scabbing thickness, perforation thickness, and the penetration depth have been obtained both experimentally and analytically $[2,3]$. A comprehensive review of the empirical formulae established by Kennedy [1],
Amirikian [4], ACE [5, 6], NDRC [7, 8], and Chang [9] was published by $\mathrm{Li}$ et al. [3]. Other empirical formulae were proposed by Young [10], BLZ [11], Bernard and Creighton [12], UMIST [13], and Zhou [14].

These formulae provide the basic references for engineering design. However, it should be noted that the majority of them were obtained via dimensional analysis of experimental results. Some dimensionless parameters in these formulae require further physical interpretation. And the dimension mismatch in some formulae also requires further investigation. The analytical calculation models based on impact mechanics are another important aspect. For instance, Luk and Forrestal [15] and Forrestal and Tzou [16] used the cavityexpansion theory to establish the locked, incompressible, and linearly compressible models for concrete targets. Li and Chen [17] proposed a new calculation method for penetration depth by introducing the nondimensional impact factor and warhead shape factor. 
Wang et al. adequately considered the destruction and deformation characteristics near the penetration cavity [18] and proposed a rigid-plastic model for thin target impact [19$21]$ and internal friction $[22,23]$ and modified hydrodynamic models $[23,24]$ for thick target penetration. Furthermore, the differential surface stress method [25] and the velocity potential theory $[26,27]$ for concrete penetration are advanced by other researchers.

The above models provide a framework for the impact process study on concrete targets, but the destruction mechanisms hiding in the empirical and analytical models still require reasonable physical explanations, and the inherent regularities also need to be discovered. In this study, based on the analysis of concrete target local destruction and deformation characteristics, the similarity laws of concrete targets impacted by rigid projectiles are studied with the rigid-plastic, internal friction, and modified hydrodynamic models for a wide range of striking velocities.

\section{The Local Effect Characteristics of Concrete Targets under Impact}

The mass, momentum, and energy conservation laws are satisfied in impact process. Within the region of local deformation and destruction under impact, the mechanical energy conservation can be expressed as

$$
\int_{S} \sigma_{n} \cdot u d S+\int_{V} F \cdot u d V=\int_{V} W d V
$$

where $\sigma_{n}$ denotes the stress on surface $S$ related to volume $V$ occupied by the material, $F$ is the mass force distribution, $u$ is the displacement vector, and $W$ is the potential energy.

In general, a part of $W$ is transformed into deformation potential energy, while some of the energy is dissipated through the fracture and shearing surfaces induced by irreversible deformation, and the remaining energy is transferred into kinetic energy through the boundaries.

$W$ can be described by the principal stresses $\sigma_{1}, \sigma_{2}$, and $\sigma_{3}$ along the three perpendicular directions and the corresponding principal strains $\varepsilon_{1}, \varepsilon_{2}$, and $\varepsilon_{3}$. An arbitrary stress state with $\sigma_{1} \geq \sigma_{2} \geq \sigma_{3}$ can be separated into three states:

$$
\begin{aligned}
{\left[\begin{array}{ccc}
\sigma_{1} & 0 & 0 \\
0 & \sigma_{2} & 0 \\
0 & 0 & \sigma_{3}
\end{array}\right]=} & T\left[\begin{array}{ccc}
1 & 0 & 0 \\
0 & 0 & 0 \\
0 & 0 & -1
\end{array}\right]+\sigma_{m}\left[\begin{array}{lll}
1 & 0 & 0 \\
0 & 1 & 0 \\
0 & 0 & 1
\end{array}\right] \\
& +T\left[\begin{array}{ccc}
0 & 0 & 0 \\
0 & \mu_{\sigma} & 0 \\
0 & 0 & 0
\end{array}\right] .
\end{aligned}
$$

For the first stress state, the maximum shearing stress $T=\left(\sigma_{1}-\sigma_{3}\right) / 2$ corresponds to a state with compression in the first principal stress direction and tension in the third principal direction. The second stress state corresponds to the hydrostatic pressure $\sigma_{m}=\left(\sigma_{1}+\sigma_{3}\right) / 2$, with equal pressure in all directions. $\mu_{\sigma}=\left(2 \sigma_{2}-\sigma_{1}-\sigma_{3}\right) /\left(\sigma_{1}-\sigma_{3}\right)$ is the Lode parameter, and the third stress state shows the influence of $\sigma_{2}$.

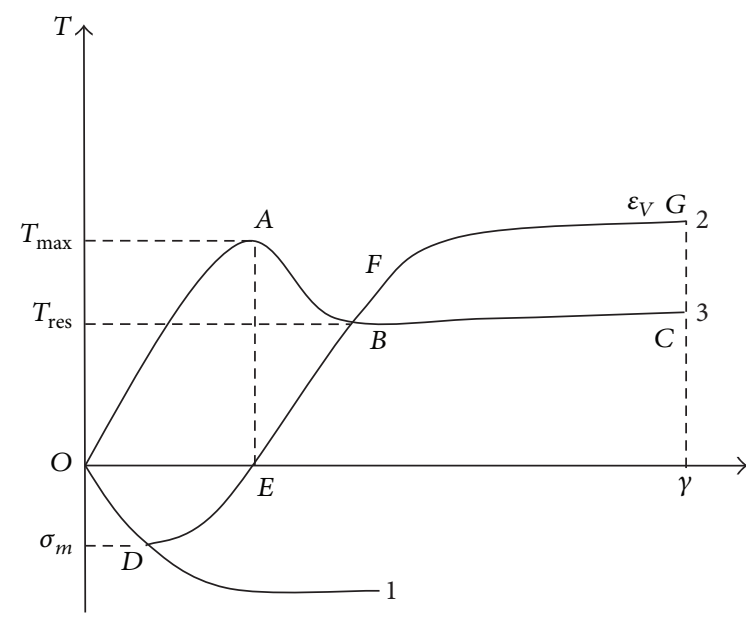

(1) Volume deformation caused by hydrostatic pressure

(2) Volume deformation caused by hydrostatic pressure and shearing stress

(3) Shearing deformation caused by shearing stresss

FIGURE 1: The shearing deformation and volume deformation with shearing stress.

Therefore, any triaxial stress state can be described with $T$, $\sigma_{m}$, and $\mu_{\sigma}$. And impact problems can be divided into typical generalized shearing and generalized compression states according to the initial impact and boundary conditions.

2.1. Generalized Shearing State. The concrete deformation characteristics are shown in Figure 1 [11]. Figure 1 can be used to study the material's response for the generalized shearing state. In Figure $1, T_{\max }$ is the maximum shear stress, $\gamma=\varepsilon_{1}-\varepsilon_{3}$ is the principal shear strain, and $\varepsilon_{V}=\varepsilon_{1}+\varepsilon_{2}+\varepsilon_{3}$ is the volume strain.

Furthermore, in Figure 1, the segment $O A$ corresponds to the elastic deformation zone under impact. The material deformation does not violate the deformation harmony condition, and in the reversible deformation state the material deformation is mainly related to elastic, viscous, and frictional properties. If the load on material increases, despite the decrease in cohesion, the strength rises due to the internal friction increase. The shearing strength limit increases with the hydrostatic pressure acting on the shearing surface. This reflects the internal friction increasing. $W$ determines the elastic energy stored in the material which is separated by the surface, and the energy storage shows a volume characteristic.

In segment $A B$, after the maximum shearing stress, the material enters the irreversible deformation state, and the surfaces of local shearing deformation appear and expand. The material is divided into blocks or pieces with certain dimensions, and the boundary conditions are satisfied through the abrupt discontinuous slipping of single surface. The residual strength of material has close relationship with the block dimension which influences the later stress and strain states. Thus, this state corresponds to the material's comminuted zone under impact. The shearing slips result in an asymmetry 
of the stress tensor, but the deformation harmony condition is still approximately satisfied because enough forces are acting on the local deformation surface. Therefore, the distributed moment appears on the discontinued lines and the material is in a quasi-continuous internal friction state.

After the peak point, with the rotation of blocks, mutual sliding occurs within the blocks; both the total strain and the shearing strain increase. Thus, the volume change and dilatation are related to the size of the destroyed blocks. The response to the change in volume is the key information. A part of $W$ is dissipated through the cracks and slip lines, which are irreversible deformations, and the energy dissipation shows superficial characteristics. The residual energy is transformed into kinetic energy through the boundary and the transferred kinetic energy shows a volume characteristic.

2.2. Generalized Compression State. Concrete and rock penetration experiments [11, 18] showed that the stress wave near the penetration cavity has the shape shown in Figure 2. The duration time of the material's positive movement is approximately one order longer than that taken to reach the maximum velocity; that is, $t_{+} / t_{-} \approx 0.1 \sim 0.2$. The higher stress and the rapid velocity change occur only in a certain narrow zone; the stress wave is almost identical to a shock wave and exhibits "short wave" properties. The difference between short wave and shock wave is the lateral deformation. For a short wave, the material moves with tiny lateral displacement, and the shearing and volume deformation are of the same order of magnitude. Therefore, the boundary of the maximum particle velocity at a certain time can be regarded as the wave front, and the following equation is satisfied:

$$
-\sigma_{r}=\rho_{0} c_{0} v_{p}, \quad v_{p}=c_{0} \varepsilon_{r},
$$

where $\sigma_{r}$ is the radial stress, $\rho_{0}$ is the density, $c_{0}$ is the longitudinal wave speed, $v_{p}$ is the particle velocity, and $\varepsilon_{r}$ is the radial strain. And, as Figure 2 shows, although the radial stress decreases after its maximum $\sigma_{r \max }$, the radial displacement increases until it is equal to the maximum $w_{\max }$. This means that the work is dissipated in order to overcome the internal friction.

Figure 3 shows the responses of columniform test specimen under uniaxial compression. The wall $A A_{1} B B_{1}$ of cylinder in Figure 3(a) is rigid and those in Figures 3(b) and $3(\mathrm{c})$ are confined. Figure 3(d) shows the unconfined uniaxial compression. In Figure 3, $K$ is the bulk modulus, $G$ is the shearing modulus, $\sigma_{r}$ is the radial stress, $\sigma_{\theta}=\sigma_{\varphi}$ are the circumferential stresses, $\varepsilon_{r}, \varepsilon_{\theta}$, and $\varepsilon_{\varphi}$ are the radial and circumferential strains, $\nu$ denotes Poisson's ratio, and $\tau_{s}$ is the shearing strength. Controlled by the impact velocity and the boundary conditions, the material's response has four states shown as Figure 3.

Figure 3(a) corresponds to segment $O A$ in Figure 1, and the material is in one-dimensional strain state, which can be regarded as uniaxial compression with rigid lateral constraint. The friction between particles is high enough and the cohesion has not been destroyed. Therefore, under compression, the material deformation is elastic and

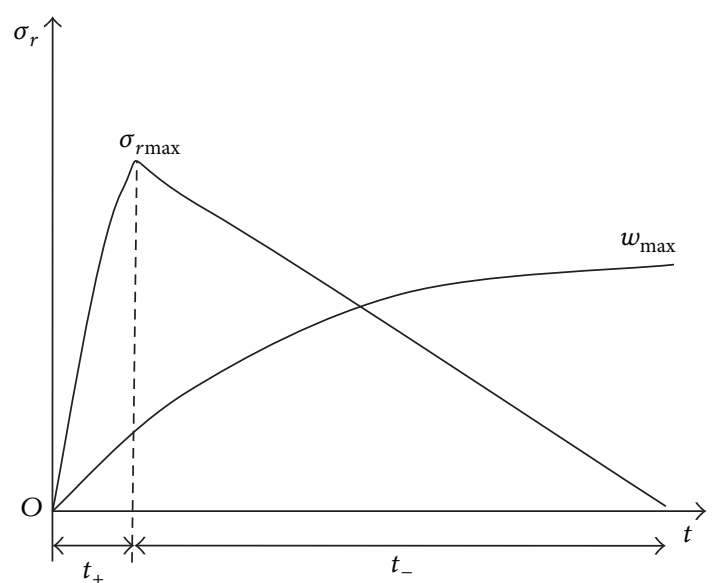

FIgURE 2: The stress wave and deformation of material near penetration cavity $[11,18,23]$.

the longitudinal wave speed is the propagation speed of small perturbations. With Hook's law, the stress and strain can be yielded as Figure 3(a).

Figure 3(b) corresponds to segment $A B$ in Figure 1, and the cohesion between the material particles is destroyed. However, the friction cannot be ignored, and the material is in a quasi-continuous internal friction state. MohrCoulomb criterion can be used and the longitudinal wave speed decreases from the small perturbation propagation speed. The material can deform laterally because of the finite constraint by the wall $A A_{1} B B_{1}$. But $\varepsilon_{\theta}$ and $\varepsilon_{\varphi}$ are very small compared with $\varepsilon_{r}$. Therefore, $\varepsilon_{r}, \varepsilon_{V}$, and $\gamma_{\max }$ are almost the same.

If the load enhances in Figure 3(b), the material response changes into Figure 3(c) which corresponds to segment $B C$ in Figure 1. In Figure 3(c), the friction between materials is almost constant and the three stresses $\sigma_{r}, \sigma_{\theta}$, and $\sigma_{\varphi}$ are almost the same. The friction between material particles is so small that it can be ignored. The longitudinal wave speed decreases to the bulk sound speed. This condition can be regarded as a fluid-like state.

Therefore, the stress state of the material changes from an elastic state to an internal friction state and eventually reaches a fluid-like state, and the deformation is the result of the gradual disappearance of the local shearing stability on multiple stages and levels. The energy near the penetration cavity is mainly dissipated because of internal friction with lateral constraint and the energy for material destruction is only a small fraction. Therefore, the stress state changes and the internal friction should be taken into account in penetration analysis. Because of the internal friction, when the material changes from elastic to plastic, it does not change to an ideal plastic state as a metal and changes to an obviously enhanced plastic state instead [11]. During the material conversion process from a state with internal friction to a fluid-like state, the longitudinal wave speed decreases from the small perturbation propagation speed to the sound speed. 


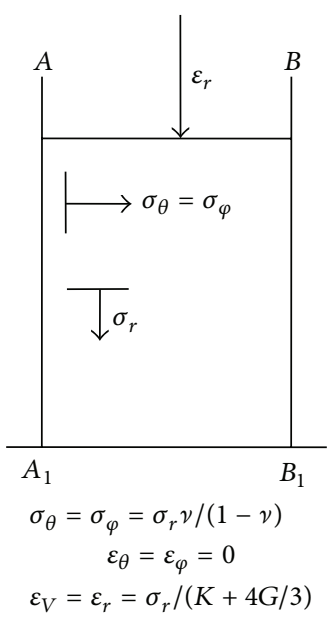

(a) Elastic state with rigid constrained lateral side

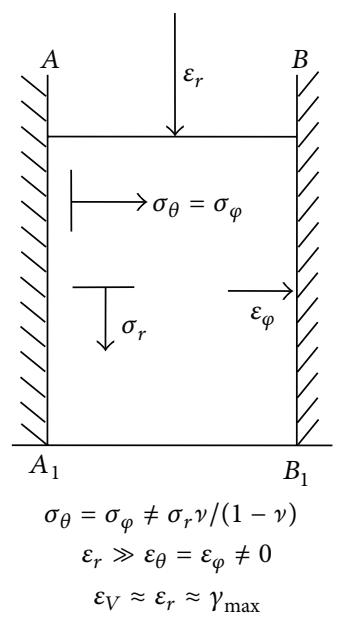

(b) Internal friction state with confined lateral side

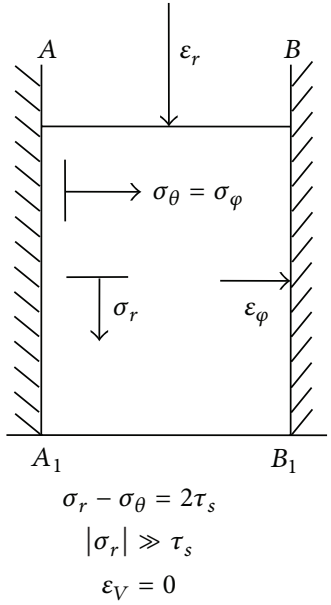

(c) Fluid-like state with confined lateral side

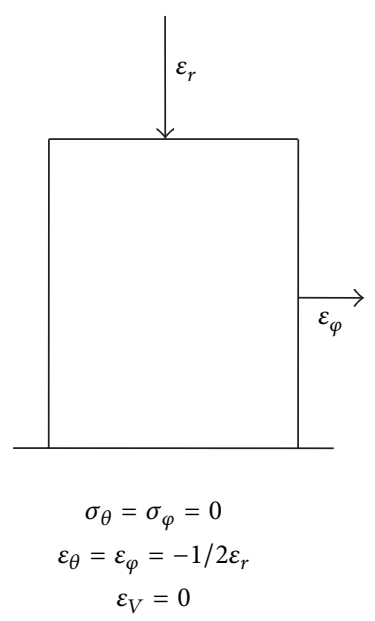

(d) Unconfined uniaxial compression

FIgURE 3: The material deformation under compression.

Figure 3(d) represents the critical state under uniaxial compression without constraint. The material has enough time to deform laterally, and severe volume compression is impossible, which is similar to the deformation state of a concrete target with finite thickness impacted at low velocity [11]. The target's free surface provides the precondition for using the incompressibility condition and rigid-plastic model.

\section{Engineering Calculation Models for Different Impact Conditions}

3.1. Rigid-Plastic Model for Thin Target Impacted at Low Velocities. For the thin target under impact, overall and local damage effects will occur. This study focused on the local ones, and the velocity varying from $30 \mathrm{~m} / \mathrm{s}$ to $150 \mathrm{~m} / \mathrm{s}$ was regarded as the low velocity considering both the engineering status quo and striking threats. For structures with finite thickness in a state as shown in Figure 3(d), impact occurs with free surfaces, and severe material volume compression cannot occur. For large deformation, the elastic component occupies a much smaller part than the plastic one, and the material near penetration cavity is in a local plastic state. Therefore, the material can be supposed as rigid-plastic and incompressible.

3.1.1. The Ultimate Resistance on Projectile. Wang et al. [19, 20] and Chen et al. [21] considered the characteristics of thin target impact and regarded the material as incompressible and rigid-plastic in order to obtain the permissive dynamic velocity field in the dynamic process by utilizing the virtual work principle and ultimate load theorem. The permissive dynamic velocity fields of flat-nose projectile impact for penetration, scabbing, and perforation are as Figure 4 shows. With the permissive dynamic velocity fields, the ultimate resistances on the projectile for penetration, scabbing, and perforation are obtained as follows:

$$
\begin{aligned}
& \frac{P^{k}}{\tau_{s}}=2 \pi-1+\pi \sqrt{2 \frac{h}{d}+\frac{1}{5},} \\
& \frac{P^{k}}{\tau_{s}}=\pi\left(\frac{L}{d}-\frac{h}{d}\right)+1, \\
& \frac{P^{k}}{\tau_{s}}=4\left(\frac{L}{d}-\frac{h}{d}\right),
\end{aligned}
$$

where $P^{k}$ is the normal component of the ultimate resistance on unit area of the projectile nose, $L$ is the target thickness, $h$ is the penetration depth, and $d$ is the projectile shank diameter.

Equation (4a) shows the ultimate resistance $P^{k}$ for penetration depth $h$ in semi-infinite target impact. Equations (4b) and (4c) are the ultimate resistances for scabbing and perforation in impact on the targets with penetration depth $h$, respectively. With (4a), (4b), and (4c), the change trend of $P^{k} / \tau_{s}$ is calculated by setting $L / d$ as 4.5 and 1.5 , as shown in Figure 5. Each ultimate resistance curve satisfies the corresponding permissive dynamic velocity field.

As Figure 4(a) shows, if the impact velocity is low, the target material in region IV does not move and the target material in regions I, II, and III move. However, the material in region I moves along the projectile velocity direction and that in region II begins changing the moving direction. At last the material in region III moves along the opposite direction of projectile velocity. With the impact velocity increasing, the target rear surface starts to influence the impact process and the target material in region IV of Figure 4(a) begins moving, and the target material does not move along negative direction of $z$-axis any more. The material in region II moves along positive directions of $z$-axis and $r$-axis. Therefore, Figure 4(a) changes into Figure 4(b) and scabbing just happens if (4a) and (4b) are equal as the intersection point $A$ of curves 1 


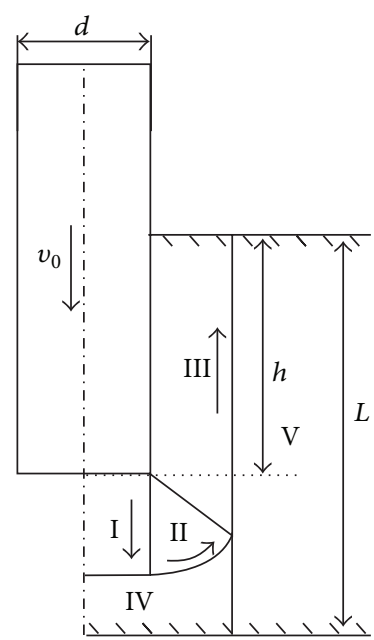

(a) Penetration velocity field

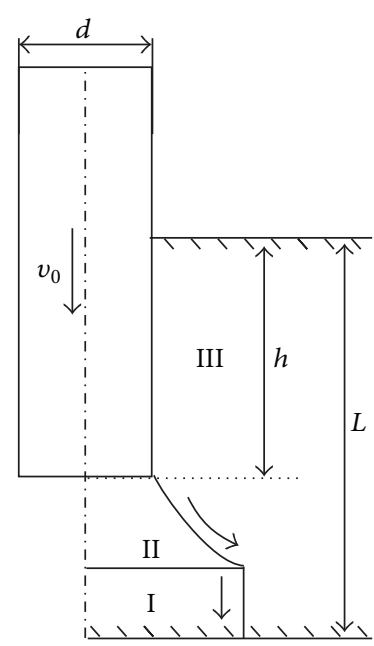

(b) Scabbing velocity field

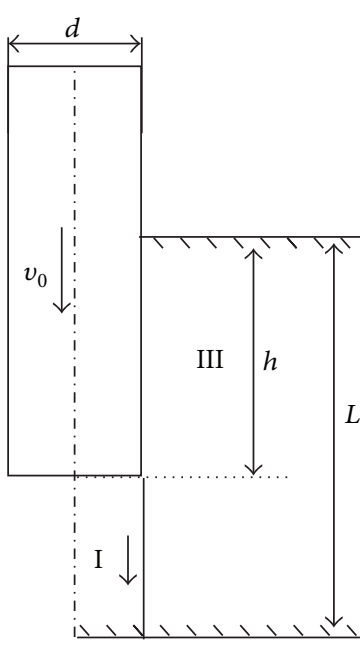

(c) Perforation velocity field

Figure 4: Permissive velocity fields of target under low velocity impact.

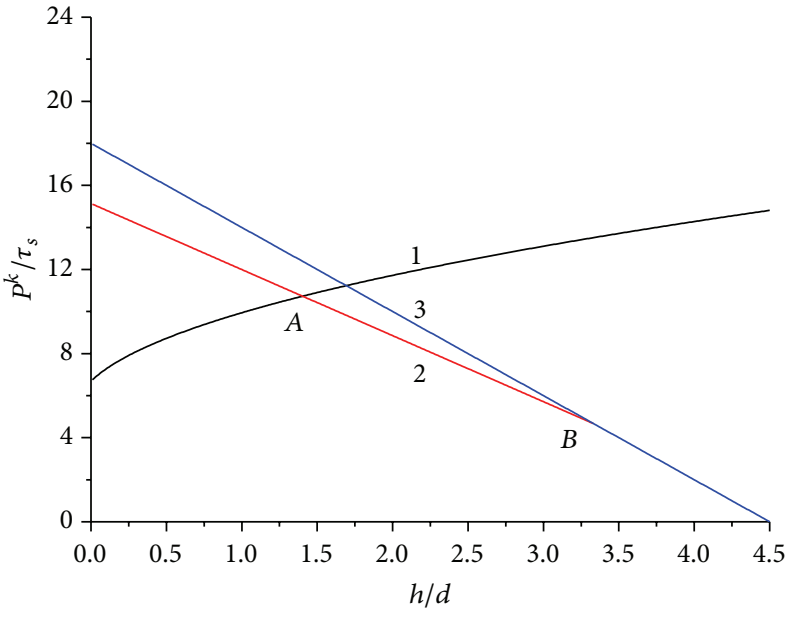

(1) Penetration velocity field

(2) Scabbing velocity field

(3) Perforation velocity field

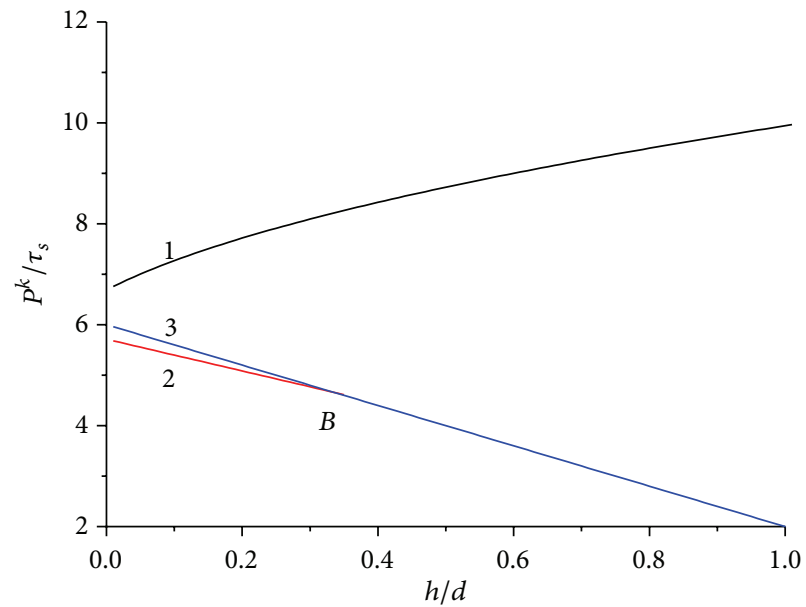

(1) Penetration velocity field

(2) Scabbing velocity field

(3) Perforation velocity field

FIGURE 5: The resistance curves of concrete target impacted by flat-nosed projectile.

and 2 in Figure 5(a) shows. This means on the left side of intersection point $A$ scabbing does not happen and on the right side of it scabbing appears. In this condition, the critical scabbing thickness $h_{s}$ can be obtained.

With the impact velocity increasing, the material of region II in Figure 4(b) does not move along positive direction of $r$-axis any more, and it moves only along the positive direction of $z$-axis. Therefore, Figure 4(b) changes into Figure 4(c) and perforation just happens if (4b) and (4c) are equal as the intersection point $B$ of curves 2 and 3 in Figures 5(a) and 5(b) shows. This means on the left side of intersection point $B$ perforation does not happen and on the right side of it perforation appears. In this condition, the critical perforation thickness $h_{p}$ can be obtained. In Figure 5(b), the curves 1 and 2 do not intersect, which means that penetration will not occur because the target thickness is too small. Therefore, for this condition, only scabbing and perforation appear at the same time.

3.1.2. The Critical Scabbing and Perforation Thicknesses. The normal resistance on projectile nose $P$ can be calculated with the following equation:

$$
P=\frac{1}{4} \pi d^{2} P^{k}
$$

The differential equation for the motion of projectile is

$$
m \frac{d^{2} h}{d t^{2}}=-P
$$

where $m$ is the projectile mass and $t$ is the time. 
TABLE 1: Tai's experimental parameters and the predicted results with (10a), (10b), and (10c).

\begin{tabular}{|c|c|c|c|c|c|c|c|}
\hline \multirow[b]{2}{*}{ Test number } & \multirow[b]{2}{*}{$\begin{array}{c}f_{c} \\
(\mathrm{MPa})\end{array}$} & \multirow[b]{2}{*}{$\begin{array}{c}f_{\text {st }} \\
(\mathrm{MPa})\end{array}$} & \multirow[b]{2}{*}{$\begin{array}{c}\tau_{s} \\
(\mathrm{MPa})\end{array}$} & \multirow[b]{2}{*}{$\begin{array}{c}v_{0} \\
(\mathrm{~m} / \mathrm{s})\end{array}$} & \multirow[b]{2}{*}{$\begin{array}{c}L \\
(\mathrm{~mm})\end{array}$} & \multicolumn{2}{|c|}{ Damage status } \\
\hline & & & & & & Test results & $\begin{array}{l}\text { Equation } \\
\text { (10a), (10b), and } \\
\text { (10c) results }\end{array}$ \\
\hline NC-F0-1 & 25 & 2.6 & 4.0 & 27.0 & 50 & Scabbing & Scabbing \\
\hline NC-F0-2 & 25 & 2.6 & 4.0 & 35.7 & 50 & Perforation & Scabbing \\
\hline NC-F0-3 & 25 & 2.6 & 4.0 & 56.8 & 50 & Perforation & Perforation \\
\hline NC-F2-1 & 25.2 & 3.1 & 4.4 & 41.7 & 50 & Scabbing & Scabbing \\
\hline NC-F2-2 & 25.2 & 3.1 & 4.4 & 56.8 & 50 & Perforation & Perforation \\
\hline NC-F2-3 & 25.2 & 3.1 & 4.4 & 64.1 & 50 & Perforation & Perforation \\
\hline RPC-F1-1 & 175.3 & 13.8 & 24.6 & 58.2 & 50 & Scabbing & Scabbing \\
\hline RPC-F1-2 & 175.3 & 13.8 & 24.6 & 76.0 & 50 & Scabbing & Scabbing \\
\hline RPC-F1-3 & 175.3 & 13.8 & 24.6 & 104.0 & 50 & Scabbing & Scabbing \\
\hline RPC-F2-1 & 178.3 & 21.9 & 31.2 & 76.0 & 50 & Scabbing & Scabbing \\
\hline RPC-F2-2 & 178.3 & 21.9 & 31.2 & 85.0 & 50 & Scabbing & Scabbing \\
\hline RPC-F2-3 & 178.3 & 21.9 & 31.2 & 104.0 & 50 & Scabbing & Scabbing \\
\hline RPC-F5-3 & 192.8 & 31.6 & 39 & 58.5 & 50 & Scabbing & Scabbing \\
\hline RPC-F5-3 & 192.8 & 31.6 & 39 & 78.1 & 50 & Scabbing & Scabbing \\
\hline RPC-F5-3 & 192.8 & 31.6 & 39 & 104.1 & 50 & Scabbing & Scabbing \\
\hline
\end{tabular}

The initial boundary condition is

$$
\begin{aligned}
\left.h\right|_{t=0} & =0 \\
\left.\frac{d h}{d t}\right|_{t=0} & =v_{0}
\end{aligned}
$$

where $v_{0}$ is the impact velocity.

With (4a), (4b), (4c) (5), (6), (7a), and (7b), the critical scabbing and perforation thickness can be yielded. For (4a), (4b), and (4c), it is found that if $L / d \leq 1.82$ the curves 1 and 2 have no intersection point and scabbing and perforation appear together. Therefore, the intersection point $B$ is used to calculate the critical perforation thickness. With (4b), (5), and (6), the following equation is yielded:

$$
\frac{m}{d} \frac{d^{2} h}{d t^{2}}=-\frac{\pi d^{2} \tau_{s}}{4}\left[\pi\left(\frac{L}{d}-\frac{h}{d}\right)+1\right] .
$$

According to (7a) and (7b) and the intersection point B's condition, it can be obtained that

$$
\begin{aligned}
\frac{L}{d}= & 0.638 I^{0.5} \tanh \left(\sqrt{\frac{\pi \tau_{s} d^{2}}{m} t}\right) \\
& +1.48 \operatorname{sech}\left(\sqrt{\frac{\pi \tau_{s} d^{2}}{m} t}\right)-0.318, \\
I= & \frac{m v_{0}^{2}}{\tau_{s} d^{3}} .
\end{aligned}
$$

If $L / d \leq 1.82$, scabbing and perforation happen together; therefore the critical perforation thickness is concerned.
The extremum of $L / d$ can be calculated to get the critical perforation thickness as

$$
\frac{h_{p}}{d}=0.64\left(\frac{m v_{0}^{2}}{\tau_{s} d^{3}}+5.3824\right)^{0.5}-0.32, \quad \frac{L}{d} \leq 1.82
$$

If $L / d \geq 1.82$, the critical scabbing and perforation thicknesses can be obtained with (4a), (4b), (4c), (5), (6), (7a), and $(7 \mathrm{~b})$ by the same method above. Consider

$$
\begin{aligned}
& \frac{h_{s}}{d}=1.28\left(\frac{m v_{0}^{2}}{\tau_{s} d^{3}}+15.3\right)^{0.5}-3.2, \quad \frac{L}{d}>1.82, \\
& \frac{h_{p}}{d}=0.75\left(\frac{m v_{0}^{2}}{\tau_{s} d^{3}}+15.3\right)^{0.5}-1.76, \quad \frac{L}{d}>1.82 .
\end{aligned}
$$

3.1.3. Comparison with Experimental Results. Tai [31] performed impact experiments on reactive powder concrete (RPC) and normal concrete (NC) with flat-nosed projectiles. The mass, diameter, and length of the projectile are $0.297 \mathrm{~kg}$, $25 \mathrm{~mm}$, and $75 \mathrm{~mm}$, respectively. The target dimensions, compositions, and the results predicted with (10a), (10b), and (10c) are given in Table 1 , where $\tau_{s}=0.5 \sqrt{f_{c} f_{\text {st }}}$. It is found that the results obtained using (10a), (10b), and (10c) are in good agreement with the experimental results (except for test NC-F0-2), suggesting that, in general, the rigid-plastic model can be used to predict the damage caused by the impact of a projectile on a thin concrete target at low velocities with a high degree of accuracy. 


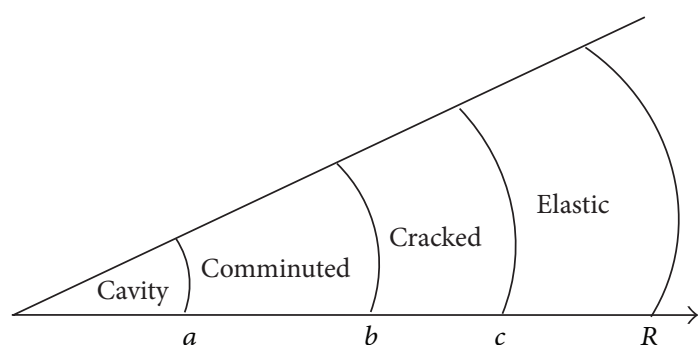

FIGURE 6: The destruction and deformation of target material in penetration.

3.2. Internal Friction Model for Thick Target Impacted at Intermediate Velocities. For a concrete target, if the condition $v / c_{0}<0.2$ is satisfied, the material near penetration cavity is in an internal friction state [11,22] indicated in Section 2.2. Therefore, $150 \mathrm{~m} / \mathrm{s}<v_{0}<0.2 c_{0}$ can be regarded as the intermediate velocity range. For thick target under impact, the material's destruction and deformation states can generally be divided into the comminuted, cracked, and elastic ones $[11,15,16,22]$ as shown in Figure 6.

3.2.1. The Resistance on Projectile Nose. The short wave in the comminuted zone represents the fastest part of the stress wave and reflects the compressibility and irreversible deformation of the material $[11,22]$ according to the following formula:

$$
-\frac{d \sigma_{r}}{d \varepsilon_{V}}=\rho_{0} c_{0}^{2}\left[1+l\left(\varepsilon_{V}\right)\right],
$$

where $l\left(\varepsilon_{V}\right)$ is the polynomial of $\varepsilon_{V}$ with $\left|l\left(\varepsilon_{V}\right)\right| \ll 1$ corresponding to the weak linear characteristic of (11).

With (3) and the mass conservation law, we obtain

$$
\varepsilon_{V}(r, t)=\frac{\dot{a}}{c_{0}}\left(\frac{a}{r}\right)^{k},
$$

where $k$ is the shear dilatation parameter, which is in the range from 1.6 to 1.8 for concrete and hard rock [11, 22], and $r$ is the radial Euler coordinate.

With (11) and (12), the general solution of radial stress in the comminuted zone is

$$
-\sigma_{r}^{p}=\rho_{0} c_{0} \dot{a}\left(\frac{a}{r}\right)^{k}+\frac{l\left(\varepsilon_{V}\right)}{2} \rho_{0} \dot{a}^{2}\left(\frac{a}{r}\right)^{2 k}+\rho_{0} c_{0}^{2} C,
$$

where $\sigma_{r}^{p}$ is the radial stress in comminuted zone and $C$ is the function to be ascertained.

In the cracked zone, the circumferential stress is zero. And because the dynamic properties of material in the cracked zone have potty influence on the solution, the inertia effect can be ignored and the equation of momentum conservation can be written as

$$
\frac{\partial \sigma_{r}}{\partial r}+\frac{2 \sigma_{r}}{r}=0 .
$$

On the boundary of the cracked and comminuted zones $(r=b)$, Tresca criterion is satisfied. Consider

$$
\sigma_{\theta}-\sigma_{r}=2 \tau_{s} .
$$

With (14) and (15), the radial stress $\sigma_{r}^{f}$ in the cracked zone is

$$
-\sigma_{r}^{f}=\frac{2 \tau_{s} b^{2}}{r^{2}} .
$$

The radial stress on the boundary of the cracked and comminuted zones is continuous which means (13) and (16) are equal if $r=b$. Therefore, function $C$ is obtained as

$$
C=\frac{2 \tau_{s}-\rho_{0} a_{0} \dot{a}(a / b)^{k}-\left(l\left(\varepsilon_{V}\right) / 2\right) \rho_{0} \dot{a}^{2}(a / b)^{2 k}}{\rho_{0} a_{0}^{2}} .
$$

Combining (13) and (17) and setting $r=a$, the cavity pressure $\sigma_{r}^{a}$ can be obtained as

$$
\begin{aligned}
& \sigma_{r}^{a}=\lambda_{s}+\lambda_{d} \rho_{0} c_{0} \dot{a}+\lambda_{e} \rho_{0} \dot{a}^{2}, \\
& \lambda_{s}=2 \tau_{s}, \\
& \lambda_{d}=1-\left(\frac{a}{b}\right)^{k} \\
& \lambda_{e}=\frac{l\left(\varepsilon_{V}\right)}{2}\left[1-\left(\frac{a}{b}\right)^{2 k}\right] .
\end{aligned}
$$

If $a / b$ is known, the resistance on projectile nose can be calculated.

3.2.2. Calculation of $a / b$. In the elastic zone, the following equations are satisfied:

$$
\begin{aligned}
\frac{\partial \sigma_{r}}{\partial r}+\frac{2\left(\sigma_{r}-\sigma_{\theta}\right)}{r} & =0, \\
\frac{\sigma_{r}-\sigma_{\theta}}{2} & =G\left(\varepsilon_{r}-\varepsilon_{\theta}\right), \\
\sigma_{r}(R) & =0 .
\end{aligned}
$$

The material can be regarded as incompressible in the elastic zone:

$$
\frac{\partial w}{\partial r}+2 \frac{w}{r}=0,
$$

where $w$ is the radial displacement.

Combining (19a), (19b), and (20), the radial stress in the elastic zone $\sigma_{r}^{e}$ is obtained as

$$
-\sigma_{r}^{e}=4 G D\left(\frac{1}{r^{3}}-\frac{1}{R^{3}}\right) .
$$

The radial stress on the boundary of elastic and cracked zone is continuous which means (16) and (21) are equal if $r=$ $c$. With this condition, $D$ is obtained:

$$
D=\frac{\tau_{s} b^{2} c}{2 G\left[1-(c / R)^{3}\right]} .
$$

With (20) and (22), the displacement of elastic zone boundary can be calculated:

$$
w^{e}(r=c)=\frac{\tau_{s} b^{2}}{2 c G\left[1-(c / R)^{3}\right]} .
$$


With the incompressible condition $\left(r^{3}-a^{3}=(r-w)^{3}\right)$, the displacements in comminuted and cracked zones can be obtained as

$$
w^{p, f}(r)=\frac{a^{3}}{3 r^{2}}
$$

On the boundary of the elastic and cracked zones $(r=c)$, the displacement is continuous, and, with (23) and (24), the following equation is yielded:

$$
\frac{a^{3}}{3 c^{2}}=\frac{\tau_{s} b^{2}}{2 c G\left[1-(c / R)^{3}\right]} .
$$

In the cracked zone, because the energy for plastic deformation can be ignored, the energy for crack growth $U_{1}$ is mainly the elastic potential energy which is half the work from outside force. Consider

$$
U_{1}=\frac{1}{2} 4 \pi b^{2}\left|\sigma_{r}^{p}(r=b)\right| \Delta w(r=b) .
$$

The whole surface energy used for new surface forming in crack growth $U_{2}$ is

$$
\begin{gathered}
U_{2}=2 n c \Delta c \gamma_{B}, \\
n=6 \pi,
\end{gathered}
$$

where $\gamma_{B}$ is the surface energy for unit area and $n$ is the number of cracks grown.

With (25), (26), (27a), and (27b), the energy conservation condition is

$$
\frac{\partial w^{p}(r=b)}{\partial c}=\frac{n c \gamma_{B}}{2 \pi b^{2} \tau_{s}} .
$$

With (24) and (25), we obtained

$$
\frac{\partial w^{p}(r=b)}{\partial c}=\frac{\tau_{s}}{2 G} \frac{1+2(c / R)^{3}}{\left[1-(c / R)^{3}\right]^{2}} .
$$

Combining (25), (28), and (29), the following equation is yielded:

$$
\frac{1}{c^{2}} \frac{1+2(c / R)^{3}}{1-(c / R)^{3}}=\frac{3 n \gamma_{B}}{2 \pi \tau_{s} a^{3}} .
$$

If the derivative of $c$ on the left side of (30) is equal to zero, the cracked zone has the extremum. This can be regarded as the condition of cracked zone boundary. Consider

$$
\frac{\partial}{\partial c} \frac{1}{c^{2}} \frac{1+2(c / R)^{3}}{1-(c / R)^{3}}=0
$$

According to (31), it is found that

$$
\left(\frac{c}{a}\right)^{2}=\frac{4 \pi \tau_{s} a}{3 n \gamma_{B}}
$$

The fracture toughness $K_{c}$ and surface energy for unit area $\gamma_{B}$ have the relationship in condition of plane strain state as

$$
\gamma_{B}=\frac{\pi K_{c}^{2}(1-\nu)}{4 G}
$$

With (25), (32), and (33), the following equations can be yielded:

$$
\begin{aligned}
\frac{c}{a} & =\sqrt{\frac{8}{9 \pi(1-\nu) \gamma_{\max }}} \sqrt{\chi}, \\
\frac{a}{b} & =\left(\frac{64 \gamma_{\max }}{18 \pi(1-\nu)}\right)^{1 / 4} \chi^{1 / 4}, \\
\gamma_{\max } & =\frac{\tau_{s}}{G} \\
\chi & =a\left(\frac{\tau_{s}}{K_{c}}\right)^{2} .
\end{aligned}
$$

Equation (34b) reveals the scaling relationship in penetration of projectiles with different calibers. In scaling experiments, the higher the scaling parameter is, the larger the difference between the real and experimental results is. Therefore, the scaling parameter should be in a certain range in order to ensure that the scaling experiment reflects reality.

3.2.3. The Penetration Depth and Comparison. For a conicalnosed projectile, the penetration resistance $F_{s}$ can be expressed as

$$
\begin{aligned}
& F_{s}=\alpha_{s}+\beta_{s} v+\eta_{s} v^{2}, \\
& \alpha_{s}=\pi a^{2}(1+\mu \cot \theta) \cdot \lambda_{s}, \\
& \beta_{s}=\pi a^{2} \rho_{0} c_{0} \lambda_{d}(1+\mu \cot \theta) \sin \theta, \\
& \eta_{s}=\pi a^{2} \rho_{0} \lambda_{e}(1+\mu \cot \theta) \sin ^{2} \theta,
\end{aligned}
$$

where $v$ is the penetration velocity, $\mu$ is the friction coefficient, and $\theta$ is half cone angle of projectile nose.

For a concrete target, the calculation results show that the third summand on the right side of (35a) can be neglected if $v / c_{0}<0.3$. The penetration depth $h_{q}$ can be calculated with the following equation $[11,22]$ :

$$
h_{q}=\frac{m}{\beta_{s}}\left[v_{0}-\frac{\alpha_{s}}{\beta_{s}} \ln \left(1+\frac{\beta_{s}}{\alpha_{s}} v_{0}\right)\right] .
$$

In (36), the linear term of $v_{0}$ reflects the influence of the material's motion in the comminuted zone on penetration, and the logarithmic term shows the effect of material strength.

In order to validate the correctness of internal friction model, the results calculated with internal friction model will be compared with results obtained from empirical formulae, for example, the Bernard I and modified NDRC equations. The concrete target and projectile parameters are listed in 
TABLE 2: The concrete target parameters $[15,16,28-30]$.

\begin{tabular}{lccccccc}
\hline Target number & $\begin{array}{c}\rho_{0} \\
\left(\mathrm{~kg} / \mathrm{m}^{3}\right)\end{array}$ & $\begin{array}{c}f_{c} \\
(\mathrm{MPa})\end{array}$ & $\begin{array}{c}K \\
(\mathrm{GPa})\end{array}$ & $\begin{array}{c}G \\
(\mathrm{GPa})\end{array}$ & $\begin{array}{c}\tau_{s} \\
(\mathrm{MPa})\end{array}$ & $\begin{array}{c}K_{c} \\
\left(\mathrm{MN} / \mathrm{m}^{3 / 2}\right)\end{array}$ & $\begin{array}{c}c_{0} \\
(\mathrm{~m} / \mathrm{s})\end{array}$ \\
\hline T-1 & 2240 & 34.5 & 9 & 4.89 & 4.5 & 0.88 \\
T-2 & 2260 & 51 & 6.7 & 4.61 & 6.5 & 2632 \\
T-3 & 2310 & 32 & 7.5 & 4.94 & 4.5 & 0.95 \\
\hline
\end{tabular}

TABLE 3: The projectile parameters [22].

\begin{tabular}{lccc}
\hline Projectile number & $m(\mathrm{~kg})$ & $d(\mathrm{~m})$ & $l_{d}$ \\
\hline P-1 & 40.3 & 0.152 & 2.04 \\
P-2 & 447 & 0.356 & 1.94 \\
\hline
\end{tabular}

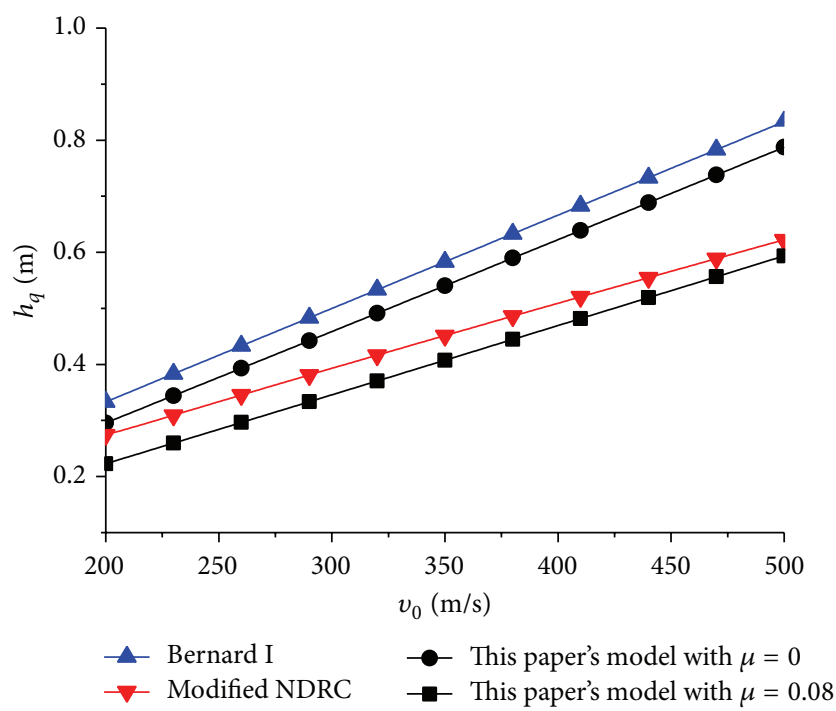

Figure 7: The predicted results for T-1 and P-1.

Tables 2 and 3 , where $l_{d}$ is the projectile nose length. The friction coefficient influences the predicted result. Forrestal [32] suggested that the friction coefficient between steel and porous rock can be ascertained as

$$
\mu= \begin{cases}0.50-0.42 \cdot\left(\frac{v}{30}\right) & v<30 \mathrm{~m} / \mathrm{s} \\ 0.08 & v \geq 30 \mathrm{~m} / \mathrm{s} .\end{cases}
$$

With (37), it is found that $\mu$ is no more than 0.08 for the velocity considered here. Therefore, 0 and 0.08 were chosen as the limiting values of $\mu$, and the predicted results for different projectile and target groups obtained with the internal friction model, the Bernard I, and modified NDRC equations are shown in Figures 7-12, respectively. As shown in Figures 7-12, the majority of results obtained with the two empirical formulae are located between the results predicted with the proposed model for $\mu=0$ and $\mu=0.08$, which means that the internal friction model describes the penetration process well. Furthermore, the slopes of the curves obtained from the internal friction model and the Bernard I formula are almost identical, especially for $\mu=0$, which strengthens the conclusion that the internal friction

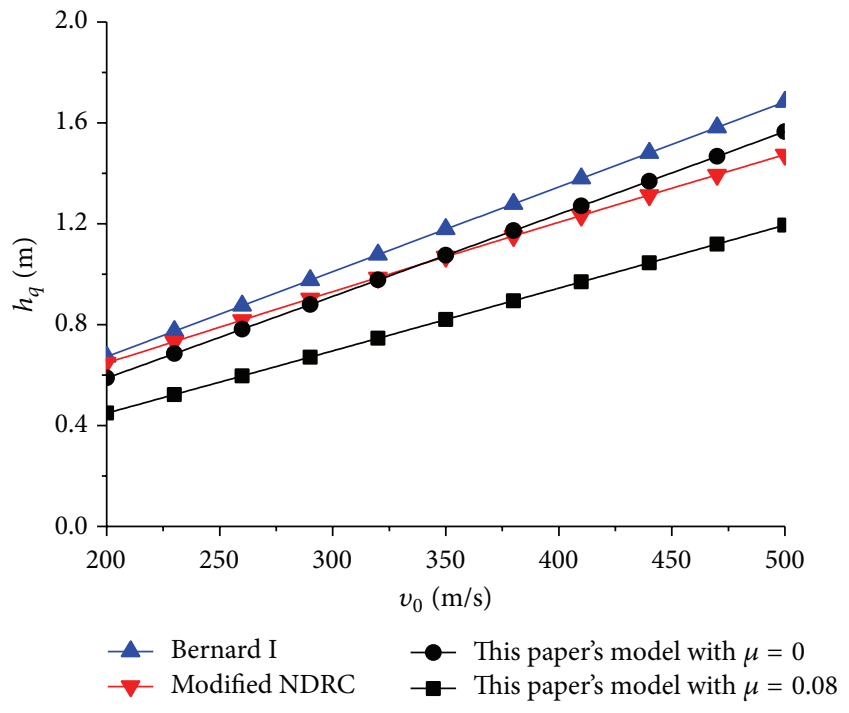

FIGURE 8: The predicted results for T-1 and P-2.

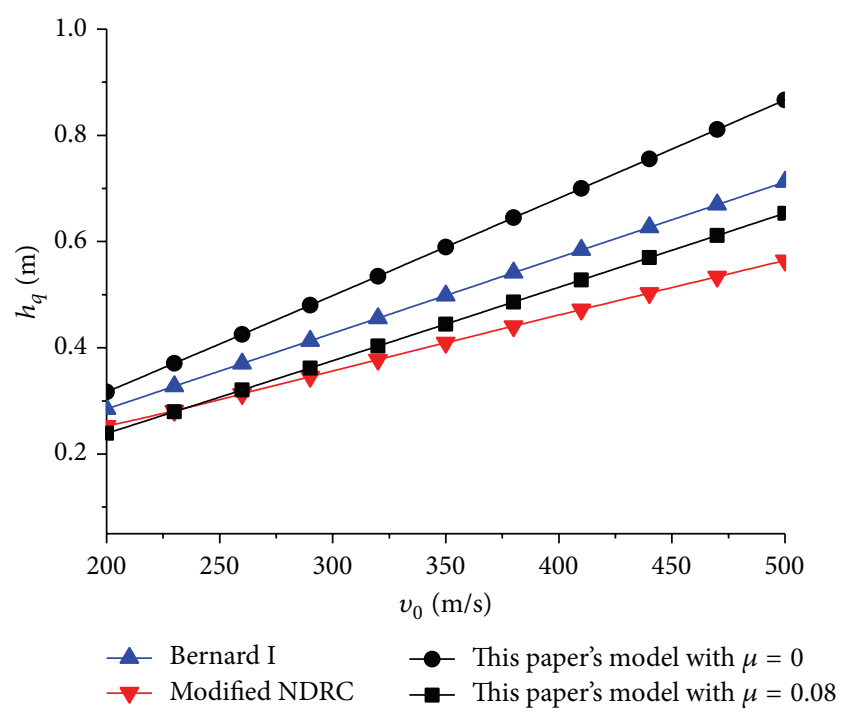

Figure 9: The predicted results for T-2 and P-1.

model can predict the penetration depth well for thick target impacted at intermediate velocities by rigid projectiles.

3.3. Modified Hydrodynamic Model for Thick Target Impacted at High Velocities. The calculation revealed that, for a concrete target, if $v / c_{0} \geq 0.2$, the comminuted material near the penetration cavity is in fluid-like state $[11,23]$, as illustrated by Figure 13. Therefore, $0.2 c_{0} \leq v_{0}<2500 \mathrm{~m} / \mathrm{s}$ was selected as 
the high velocity range, with both current and future weapon velocities considered.

For a high velocity penetration, the comminuted material can be considered as an ideal incompressible fluid with density $\rho_{0}$. If the pressure on the fluid-like boundary $\Gamma$ is equal to the target material dynamic hardness $H$ [33-36], the impulse equation and the Bernoulli equation of fluid with the continuous condition can be written as [37]

$$
\begin{aligned}
\pi\left(b^{2}-a^{2}\right) k_{c} \rho_{0} v_{\infty}^{2}-\pi b^{2} \rho_{0} v^{2} & =\pi b^{2} H-q, \\
H+\frac{\rho_{0} v^{2}}{2} & =\frac{\rho_{0} v_{\infty}^{2}}{2}, \\
\left(b^{2}-a^{2}\right) k_{c} v_{\infty} & =b^{2} v,
\end{aligned}
$$

where $k_{c}$ is the contraction coefficient of the fluid-like material, $v_{\infty}$ is the fluid-like material velocity limit, and $q$ is the penetration resistance. Using (38a), (38b), and (38c), the following equations can be obtained:

$$
\begin{aligned}
q & =\pi a^{2} H q_{0}, \\
q_{0} & =\frac{\sqrt{1+\eta^{2}}\left(\sqrt{1+\eta^{2}}-\eta\right)^{2}}{\sqrt{1+\eta^{2}}-\eta / k_{c}}, \\
\eta & =\frac{v}{\sqrt{2 H / \rho_{0}}} .
\end{aligned}
$$

Therefore, the penetration depth can be calculated according to

$$
h_{q}=m \int_{0}^{v_{0}} \frac{v}{\pi a^{2} H q_{0}} d v .
$$

From (38a), (38b), (38c), (39a), (39b), and (39c), it is clear that $k_{c}$ is the key parameter required to solve the equations. According to Slepyan [37], for this axisymmetric problem, $k_{c}$ can be directly calculated by solving the plane problem of a wedge by replacing $\pi b^{2}$ and $\pi a^{2}$ with $2 b$ and $2 a$, respectively. Then

$$
k_{c}=\frac{\xi}{1-a / b}
$$

with

$$
\begin{aligned}
\xi & =\sqrt{\frac{\eta^{2}}{\left(1+\eta^{2}\right)}}, \\
\frac{a}{b} & =\frac{\sin \theta}{\pi} \\
& \cdot \xi \int_{0}^{1}\left(\frac{1}{\omega+\xi^{\pi / \theta}}+\frac{1}{\omega+\xi^{\theta / \pi}}-\frac{2}{\omega+1}\right) \frac{1}{\omega^{\theta / \pi}} d \omega .
\end{aligned}
$$

The penetration depth is determined with (39a), (39b), (39c), (40), (41a), (41b), and (41c). However, it is impossible to obtain an analytical solution for the penetration depth, and then the numerical method is used.

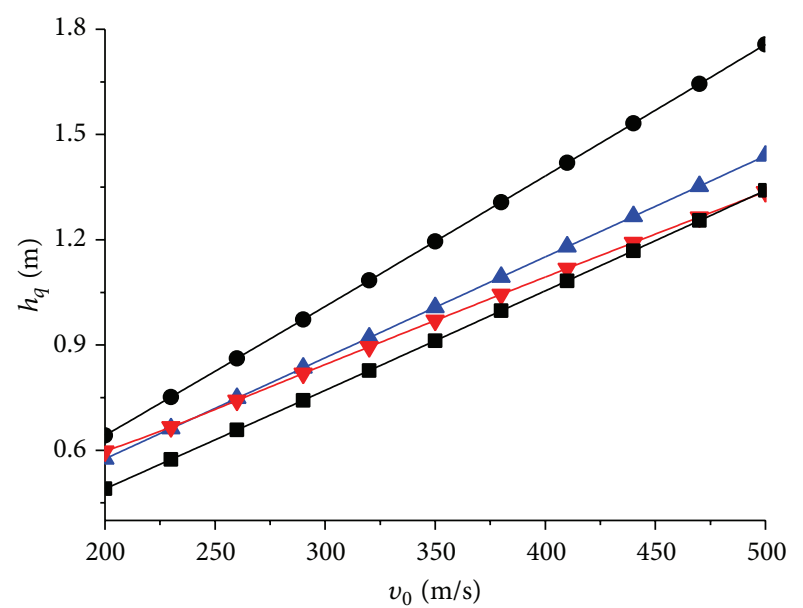

- Bernard I
$-\nabla$ Modified NDRC $\quad \longrightarrow$ This paper's model with $\mu=0$
- This paper's model with $\mu=0.08$

Figure 10: The predicted results for T-2 and P-2.

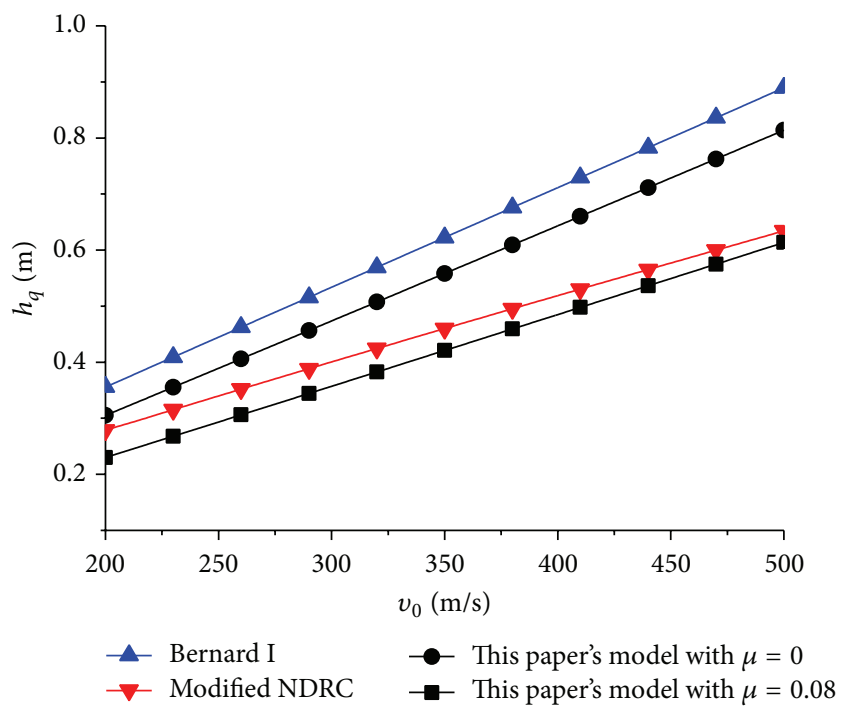

Figure 11: The predicted results for T-3 and P-1.

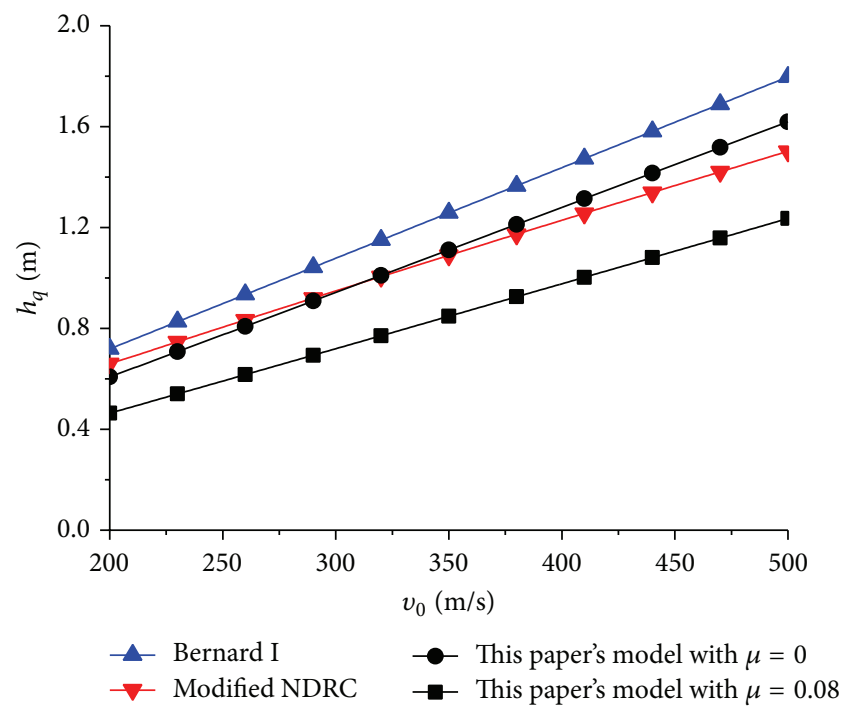

Figure 12: The predicted results for T-3 and P-2. 


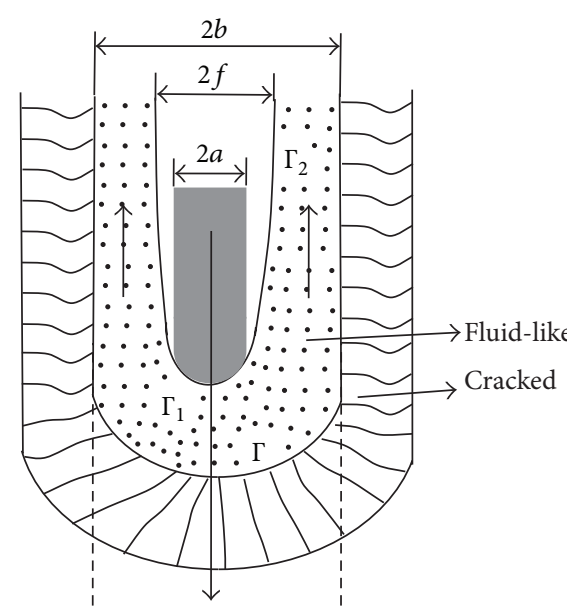

(a)

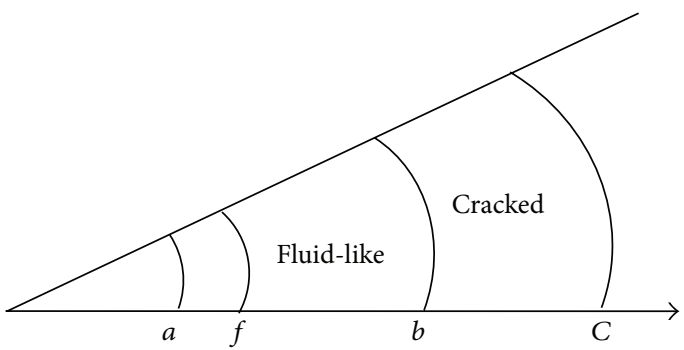

(b)

FIGURE 13: High velocity projectile penetrating concrete target.

Forrestal et al. [29] performed penetration experiments on concrete targets with $0.90 \mathrm{~kg} 4340$ steel rods whose CRH and diameter are 2 and $26.9 \mathrm{~mm}$, respectively. The projectiles were heat-treated in order to obtain a hardness of Rc 43-45, so that they remained rigid during the experiments. The target parameters are as follows:

$$
\begin{aligned}
\rho_{0} & =2310 \mathrm{~kg} / \mathrm{m}^{3}, \\
K & =7.5 \mathrm{GPa}, \\
\nu & =0.23, \\
H & =518 \mathrm{MPa}, \\
Y & =150 \mathrm{MPa} .
\end{aligned}
$$

$H$ was calculated with Wang's formula [38]:

$$
H=\frac{2 Y}{3}\left[1+\ln \frac{2 G}{Y}\right]
$$

where $Y$ is the dynamic shear strength.

In foregoing conditions, the predicted results were compared with the experimental ones and are shown in Figure 14. The predicted results are in good agreement with the experimental values for $v_{0}>500 \mathrm{~m} / \mathrm{s}$, except for case 4 . However, the experimental result obtained for case 4 is aberrant when compared with the whole range of experimental results. If $v_{0} \leq 500 \mathrm{~m} / \mathrm{s}$, the predicted penetration depths are lower than the experimental values obtained for cases 13 in Figure 14, which means that the model cannot accurately predict the penetration depths at lower velocities. Furthermore, for this concrete target, $500 \mathrm{~m} / \mathrm{s}$ is close to $0.2 c_{0}=494 \mathrm{~m} / \mathrm{s}$, which is the critical value for high velocity penetration. Therefore, it can be concluded that the modified hydrodynamic model can accurately predict the penetration depth for high velocity impact by rigid projectile.

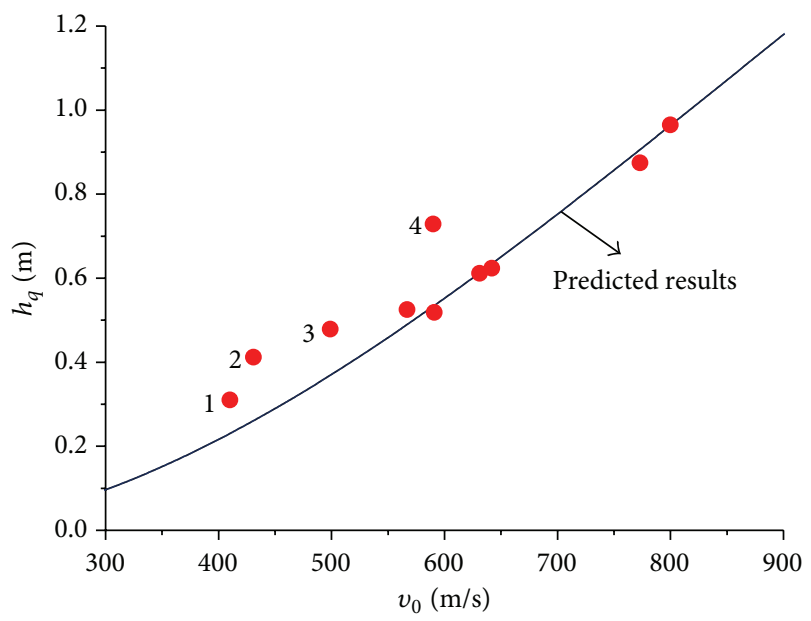

FIGURE 14: The experimental results [29] and predicted penetration depth.

\section{Similarity Laws for the Impact on a Concrete Target}

4.1. Dimensional Analysis. Dimensional analysis is the effective method in physics research and needs cogent analysis on the dimensions of main variables in the problem [39]. At present, majority of the widely used empirical formulae for concrete impact were obtained via dimensional analysis with experimental results. In different impact conditions, the main factors which influence the impact results are different. In this paper, the research object is the rigid projectile impact on a concrete target, and the relative parameters of projectile deformation are not considered. The projectile mass $m$, the diameter $d$, and the nose shape parameter $N^{*}$ are taken into account. For a concrete target, thickness $L$, material density $\rho_{0}$, shearing strength $\tau_{s}$, Young's modulus $E$, longitudinal wave speed $c_{0}$, fracture toughness $K_{c}$, and dynamic hardness $H$ are the main parameters that are considered. Furthermore, 
the impact velocity $v_{0}$ and the friction coefficient $\mu$ are also included in the analysis. Then, the penetration depth $h_{q}$, the critical scabbing thickness $h_{s}$, and the perforation thickness $h_{p}$ can be written as

$$
\begin{aligned}
& h_{q}=g_{q}\left(m, d, N^{*}, L, \rho_{0}, \tau_{s}, E, c_{0}, K_{c}, H, v_{0}, \mu\right), \\
& h_{s}=g_{s}\left(m, d, N^{*}, L, \rho_{0}, \tau_{s}, E, c_{0}, K_{c}, H, v_{0}, \mu\right), \\
& h_{p}=g_{p}\left(m, d, N^{*}, L, \rho_{0}, \tau_{s}, E, c_{0}, K_{c}, H, v_{0}, \mu\right) .
\end{aligned}
$$

Li and Chen [17] deemed that Young's modulus is not the main influence factor and found that Young's modulus is not included in the majority of the empirical formulae and ignored this parameter in their analysis. Young's modulus describes the target material's property in elastic state, while the material's property in plastic state is the main factor for penetration resistance, so it is reasonable to ignore Young's modulus.

$\mathrm{Li}$ and Chen [17] suggested that the friction coefficient between projectile and target can be ignored in rigid projectile penetration, and as the case stands, $\mu$ is difficult to measure [40]. According to (37), $\mu$ is very small, so the influence of $\mu$ can be ignored. $\tau_{s}$ and $H$ as strength representations are included in (44a), (44b), and (44c); however, they have different effects on impact. $\tau_{s}$ reveals the area character in energy transferring and has local plastic property. However, $H$ is the energy consumption per unit volume for cavity and reflects the volume character of energy consumption and is the embodiment of the material's overall plastic state and dynamical cratering. It contains much important information, such as the strain rate effect, the inertia, and strength effects [41]. Therefore, $\tau_{s}$ and $H$ should be preserved in (44a), (44b), and (44c). In thick target penetration, $L$ is not the main influence factor which controls the process, but it plays a very important role in scabbing and perforation of thin target, and the critical target thicknesses for scabbing and perforation in thin target impact are equal to the target's thickness $L$. Therefore, $L$ can be eliminated in (44a), (44b), and (44c), and it can then be rewritten as

$$
\begin{aligned}
& h_{q}=g_{q}\left(m, d, N^{*}, \rho_{0}, \tau_{s}, c_{0}, K_{c}, H, v_{0}\right), \\
& h_{s}=g_{s}\left(m, d, N^{*}, \rho_{0}, \tau_{s}, c_{0}, K_{c}, H, v_{0}\right), \\
& h_{p}=g_{p}\left(m, d, N^{*}, \rho_{0}, \tau_{s}, c_{0}, K_{c}, H, v_{0}\right) .
\end{aligned}
$$

Equations (45a), (45b), and (45c) can be transformed to

$$
\begin{aligned}
& \frac{h_{q}}{d}=g_{q}\left(\frac{m v_{0}^{2}}{d^{3} \tau_{s}}, N^{*}, \frac{\tau_{s}}{\rho_{0} c_{0} v_{0}}, \frac{\tau_{s} d^{0.5}}{K_{c}}, \frac{\tau_{s}}{H}\right), \\
& \frac{h_{s}}{d}=g_{s}\left(\frac{m v_{0}^{2}}{d^{3} \tau_{s}}, N^{*}, \frac{\tau_{s}}{\rho_{0} c_{0} v_{0}}, \frac{\tau_{s} d^{0.5}}{K_{c}}, \frac{\tau_{s}}{H}\right), \\
& \frac{h_{p}}{d}=g_{p}\left(\frac{m v_{0}^{2}}{d^{3} \tau_{s}}, N^{*}, \frac{\tau_{s}}{\rho_{0} c_{0} v_{0}}, \frac{\tau_{s} d^{0.5}}{K_{c}}, \frac{\tau_{s}}{H}\right) .
\end{aligned}
$$

In (46a), (46b), and (46c), $I=m v_{0}^{2} /\left(d^{3} \tau_{s}\right)$, and it shows the same form as the impact factors suggested by Chang [9],
TABLE 4: The parameters in (47a), (47b), and (47c) for different $I$.

\begin{tabular}{lcccccc}
\hline$I$ & $m_{s}$ & $n_{s}$ & $m_{p}$ & $n_{p}$ & $m_{s p}$ & $n_{s p}$ \\
\hline$I<1$ & 1.900 & 0.015 & 1.250 & 0.025 & 1.215 & 0.0165 \\
$1 \leq I<6$ & 1.850 & 0.200 & 1.200 & 0.180 & 1.250 & 0.2032 \\
$I \geq 6$ & 0.990 & 0.525 & 0.532 & 0.540 & 0.610 & 0.5040 \\
\hline
\end{tabular}

Reid and Wen [13], Hughes [42], Haldar and Hamieh [43], Kojima [44], and Li and Chen [17, 45]. The only difference is the fact that the uniaxial compressive strength is replaced by the shearing strength. Therefore, $I=m v_{0}^{2} /\left(d^{3} \tau_{s}\right)$ is also defined as the impact factor and indicates the ability of projectile to destroy the target. $N^{*}$ represents the influence of the projectile's nose shape on the impact. $\Psi=\tau_{s} /\left(\rho_{0} c_{0} v_{0}\right)$ shows the relationship between the shearing strength and the dynamic stress and essentially represents the energy ratio of destruction and particle motion and shows the distribution of impact energy. Therefore, it can be defined as dynamic factor. $\Lambda=\tau_{s} d^{0.5} / K_{c}$, defined as toughness factor, shows the relationship between the thickness of the comminuted and cracked zones. $Q=\tau_{s} / H$ represents the energy distribution between the local destruction and overall plastic destruction and can be defined as dynamic hardness factor. For targets consisting of the same material, impact factor is the main parameter that determines the destruction effect and can be used to guide the design of scaling experiments.

4.2. Similarity Laws in the Calculation Models for Concrete Target Impact. At present, many empirical formulae for concrete target impact were obtained by dimensional analysis and more or less show the effect of impact factor, with only slight differences in form or power [3]. To reveal the influence extent of $I, \Psi$, and $Q$ in (46a), (46b), and (46c), the similarity laws for impact on a concrete target will be studied based on the models in Section 3.

4.2.1. Similarity Laws of the Impact on a Thin Target at Low Velocities. According to the general forms given by (46b) and (46c), by curve fitting, (10a), (10b), and (10c) can be rewritten as

$$
\begin{array}{ll}
\frac{h_{s}}{d}=m_{s} I^{n_{s}}, & \frac{L}{d}>1.82, \\
\frac{h_{p}}{d}=m_{p} I^{n_{p}}, & \frac{L}{d}>1.82, \\
\frac{h_{p}}{d}=m_{s p} I^{n_{s p}}, & \frac{L}{d} \leq 1.82,
\end{array}
$$

and the parameters in (47a), (47b), and (47c) are listed in Table 4.

According to (47a), (47b), and (47c), the impact factor I is shown to be the unique factor that determines the critical scabbing and perforation thicknesses, and $\Psi, \Lambda$, and $Q$ have no influence on these critical thicknesses. The local plastic deformation state of the target material caused by the free surface brings the results above, and the rear surface of target seriously influences the impact process. The exponents of 
the impact factor are close to 0.5 for $I \geq 6$, suggesting an approximately linear relationship between the target critical thicknesses (scabbing and perforation) and impact velocity.

4.2.2. Similarity Laws of the Impact on a Thick Target at Intermediate Velocities. Calculations showed that if $0.1<v_{0} / c_{0}<$ 0.2 , the influence of the logarithmic term in (36) is below $5 \%$. Therefore, (36) can be rewritten as

$$
\begin{aligned}
& \frac{h_{q}}{d}=\frac{\lambda_{1} \lambda_{2} m v_{0}}{d^{3} \rho_{0} c_{0}}=\lambda_{1} \lambda_{2} I \cdot \Psi, \\
& \lambda_{1}=\frac{4}{\pi(1+\mu \cot \theta) \sin \theta}, \\
& \lambda_{2}=\frac{1}{1-0.9418^{k} \gamma_{\max }^{k / 4} \Lambda^{k / 8}} .
\end{aligned}
$$

Equation (48a) means that, for a certain impact velocity range, the penetration depth is approximately directly proportional to the impact velocity. This is consistent with the power of velocity term in the formulae proposed by Young [10] and BLZ [11]. Furthermore, for the penetration of a thick target at intermediate velocities, $I, \Lambda$, and $\Psi$ are the principal factors that determine the penetration process, and the material's internal friction motion in the comminuted zone is the pivotal point for penetration resistance.

4.2.3. Similarity Laws of the Impact on a Thick Target at High Velocities. As mentioned above, it is difficult to yield an analytical calculation formula for the modified hydrodynamic model. Therefore, the numerical method was applied for calculation. With the parameters given in Section 3.3, the penetration resistance and depth were calculated for different cone angles, and the results are shown in Figures 15 and 16. The resistance shows a linear relationship with $m v^{2} / d^{3} H$ for a certain penetration velocity according to the following formula:

$$
q=\frac{\pi H d^{2}}{4}\left(a_{0}+a_{1} \frac{m v^{2}}{d^{3} H}\right)
$$

where $a_{0}$ and $a_{1}$ are dimensionless parameters obtained from the curves in Figure 15. Using (40) and (49), the scaling penetration depth can then be calculated as follows:

$$
\frac{h_{q}}{d}=\frac{2}{\pi a_{1}} \ln \left(1+\frac{a_{1} I \cdot Q}{a_{0}}\right) .
$$

In (50), the scaling penetration depth is a function of the impact factor $I$ and hardness factor Q. Equation (50) further reveals that the scaling penetration depth has a logarithmic relationship with the impact factor and the hardness factor as shown in Figure 16. However, if $10 \%$ can be considered to be an acceptable fitting error, (50) can be fitted with the following function:

$$
\frac{h_{q}}{d}=m_{h}(I \cdot Q)^{n_{h}}
$$

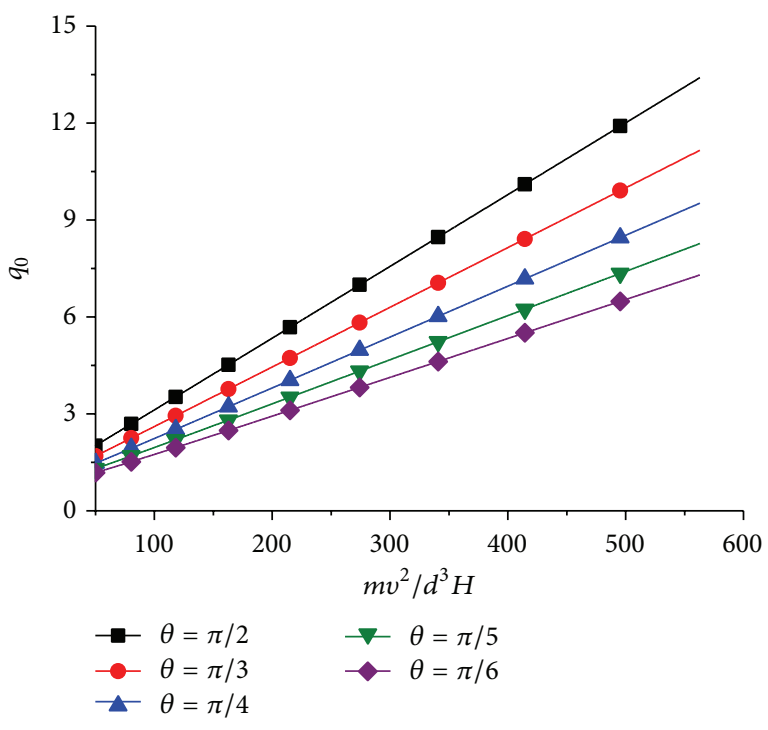

FIGURE 15: The dimensionless resistance on projectile.

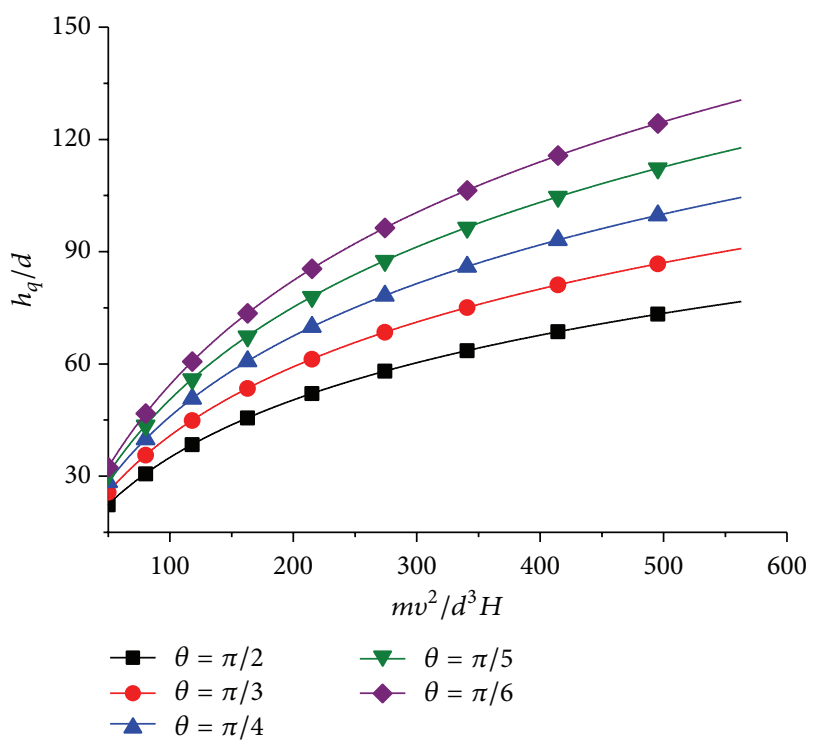

FIGURE 16: The scaling penetration depth.

where $m_{h}$ and $n_{h}$ are the curve fitting parameters. The calculation showed that $n_{h}$ is in the range from 0.3 to 0.35 , which means that there exists a $0.6-0.7$ power relationship between the scaling penetration depth and impact velocity. This is in agreement with the famous $2 / 3$ power scaling law obtained for spherical projectile experiments on metal [46] and rock [47] targets, which indicates that, for the impact of a rigid projectile on a concrete target, the $2 / 3$ power scaling law also exists. The hardness factor is determined by target material only. In contrast, the impact factor is determined by both the projectile and target. Therefore, the impact factor represents the similarity law in penetration and can be used as the controlling parameter for the design of scaling experiments. 


\section{Conclusion}

Controlled by the initial conditions, such as the target dimensions and impact velocities, the concrete target undergoes different destruction and deformation states, resulting in different impact effects and similarity laws. In this paper, the generalized shearing and compression states of the impact on a concrete target were analyzed. Based on the rigidplastic, internal friction, and modified hydrodynamic models corresponding to the destruction and deformation characteristics of the concrete advanced by the authors, the following conclusions were obtained according to dimensional analysis:

(1) For the impact on a thin target at low velocities, the impact factor is the only parameter controlling the impact. There is an approximately linear relationship between the critical thicknesses and impact velocity.

(2) For the impact on a thick target at intermediate velocities, the impact, toughness, and dynamic factors together determine the penetration behavior. There is an approximately linear relationship between the penetration depth and impact velocity.

(3) For the impact on a thick target at high velocities, the impact and hardness factors together determine the penetration behavior. There is a $2 / 3$ power relationship between the penetration depth and the impact velocity.

(4) For the impact on a thick target at different impact velocities, different distinct scaling laws exist. For impact at intermediate velocities, there is a linear relationship between the ratio of the projectile and cavity radii, and there is no relationship with the penetration velocity, which reveals the scaling conversion relationship for the impact of projectiles with different calibers on a concrete target. The higher the scaling coefficient in the experiments is, the larger the difference between the simulation and real results is. However, for impact at high velocities, there is only a relationship between the ratio of projectile and cavity radii and the penetration velocity, which means that the scaling coefficient has little influence on the simulation result for the impact of projectiles with different calibers, and therefore the scaling experiments can be flexibly designed.

\section{Nomenclature}

$\sigma_{n}: \quad$ Stress on the surface $S$ related to the

volume $V$ occupied by the material

$F$ : $\quad$ Mass force distribution

$u$ : Displacement vector

$W$ : $\quad$ Potential energy

$\sigma_{1}, \sigma_{2}, \sigma_{3}$ : Principal stresses

$\varepsilon_{1}, \varepsilon_{2}, \varepsilon_{3}$ : Principal strains

$T: \quad$ Shearing stress

$\sigma_{m}: \quad$ Hydrostatic pressure

$\mu_{\sigma}$ : Lode parameter

$T_{\max }: \quad$ Maximum shear stress $\gamma: \quad \quad$ Principal shear strain

$\varepsilon_{v}: \quad$ Volume strain

$t: \quad$ Time

$t_{+}: \quad$ Duration time of radial stress increasing

$t_{-}: \quad$ Duration time of radial stress decreasing

$\sigma_{r}: \quad$ Radial stress

$\rho_{0}: \quad$ Density

$c_{0}: \quad$ Longitudinal wave speed

$v_{p}: \quad$ Particle velocity

$\varepsilon_{r}: \quad$ Radial strain

$\sigma_{r \max }: \quad$ Maximum radial stress

$w_{\max }: \quad$ Maximum radial displacement

$K: \quad$ Bulk modulus

$G$ : $\quad$ Shearing modulus

$\sigma_{\theta}, \sigma_{\phi}: \quad$ Circumferential stresses

$\varepsilon_{\theta}, \varepsilon_{\phi}: \quad$ Circumferential strains

$v$ : Poisson's ratio

$\tau_{s}: \quad$ Shearing strength

$P^{k}$ : $\quad$ Normal component of the ultimate resistance on unit area of the projectile nose

L: $\quad$ Target thickness

$h$ : Penetration depth of thin target

$h_{s}: \quad$ Critical scabbing thickness

$h_{p}$ : Critical perforation thickness

P:

$d:$

$m: \quad$ Projectile mass

$v_{0}: \quad$ Impact velocity

$f_{c}: \quad$ Unconfined compressive strength

$f_{\text {st }}: \quad$ Split tensile strength

$v: \quad$ Penetration velocity

Ф: Integration variable in (41c)

$Y: \quad$ Dynamic shear strength

$E: \quad$ Young's modulus

$N^{*}$ : $\quad$ Projectile nose shape parameter

I: $\quad$ Impact factor

$\Psi: \quad$ Dynamic factor

$\Lambda: \quad$ Toughness factor

$l\left(\varepsilon_{v}\right): \quad$ Polynomial of $\varepsilon_{v}$

a: $\quad$ Cavity radius

b: $\quad$ Comminuted zone radius

$c: \quad$ Cracked zone radius

$R: \quad$ Elastic zone radius

$C: \quad$ Function to be ascertained in (13)

$k: \quad$ Shear dilatation parameter

$r: \quad$ Radial Euler coordinate

$\sigma_{r}^{p}: \quad$ Radial stress in comminuted zone

$\sigma_{r}^{f}$ : Radial stress in cracked zone

$\sigma_{r}^{a}$ : $\quad$ Cavity pressure

$\lambda_{s}, \lambda_{d}$, and $\lambda_{e}$ : Coefficients defined in (18a), (18b), (18c), and (18d)

$w: \quad$ Radial displacement

$\sigma_{r}^{e}: \quad$ Radial stress in elastic zone

D: $\quad$ Function to be ascertained in (21)

$w^{e}$ : Radial displacement in elastic zone

$w^{p, f}$ : $\quad$ Radial displacement in comminuted and cracked zones 


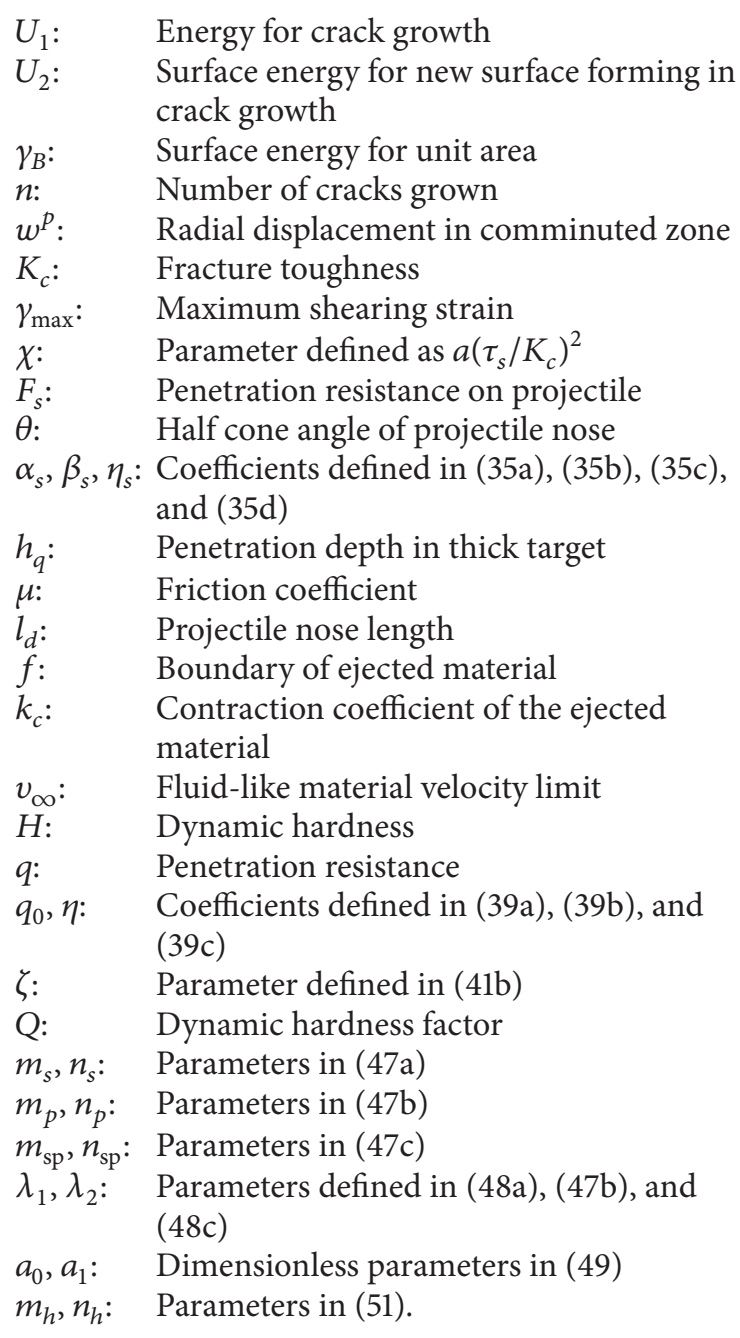

\section{Conflict of Interests}

The authors declare that there is no conflict of interests regarding the publication of this paper.

\section{Acknowledgments}

The authors gratefully acknowledge the financial supports from Program for Changjiang Scholars and Innovative Research Team in University (no. IRT13071), the Science Fund for Creative Research Group of the National Natural Science Foundation of China (Grant no. 510210001), projects supported by the National Natural Science Foundation of China (Grant nos. 51409258, 51309233, and 51378498), and project supported by State Key Laboratory for Disaster Prevention \& Mitigation of Explosion \& Impact Foundation (Grant no. DPMEIKF201301).

\section{References}

[1] R. P. Kennedy, "A review of procedures for the analysis and design of concrete structures to resist missile impact effects," Nuclear Engineering and Design, vol. 37, no. 2, pp. 183-203, 1976.
[2] X. Chen, "Advances in the penetration/perforation of rigid projectiles," Advances in Mechanics, vol. 39, pp. 316-351, 2009.

[3] Q. M. Li, S. R. Reid, H. M. Wen, and A. R. Telford, "Local impact effects of hard missiles on concrete targets," International Journal of Impact Engineering, vol. 32, no. 1-4, pp. 224-284, 2005.

[4] A. Amirikian, "Design of protective structures," Tech. Rep. NT3726, Bureau of Yards and Docks, Departement of the Navy, 1950.

[5] C. V. Chelapati, R. P. Kennedy, and I. B. Wall, "Probabilistic assessment of aircraft hazard for nuclear power plants," Nuclear Engineering and Design, vol. 19, no. 2, pp. 333-364, 1972.

[6] ACE, "Fundamentals of protective structures," Tech. Rep. AT120 AT1207821, Army Corps of Engineers, Office of the Chief of Engineers, 1946.

[7] NDRC, Effects of Impact and Explosion. Summary Technical Report of Division 2, vol. 1, National Defence Research Committee, Washington, DC, USA, 1946.

[8] R. P. Kennedy, Effects of an Aircraft Crash into a Concrete Reactor Containment Building, Holmes \& Narver, Anaheim, Calif, USA, 1966.

[9] W. S. Chang, "Impact of solid missiles on concrete barriers," ASCE Journal of the Structural Division, vol. 107, pp. 257-271, 1981.

[10] C. W. Young, "Penetration equations," SAND 97-2426, Sandia National Laboratories, 1997.

[11] Q. H. Qian and M. Y. Wang, The Impact and Explosion Effects in Rock and Soil, National Defence Industrial Press, Beijing, China, 1st edition, 2010.

[12] R. S. Bernard and D. C. Creighton, "Projectile penetration in soil and rock: analysis for non-normal impact," Tech. Rep. SL-79-15, U.S. Army Engineer Waterways Experiment Station, 1979.

[13] S. R. Reid and H. M. Wen, "Predicting penetration, cone cracking, scabbing and perforation of reinforced concrete targets struck by flat-nosed projectiles," UMIST Report ME/AM/02.01/TE/G/018507/Z, 2001.

[14] Z. Zhou, Study on the local and total response of reinforced concrete structure under low velocity impact [Ph.D. thesis], PLA University of Science and Technology, Nanjing, China, 2008.

[15] V. K. Luk and M. J. Forrestal, "Penetration into semi-infinite reinforced-concrete targets with spherical and ogival nose projectiles," International Journal of Impact Engineering, vol. 6, no. 4, pp. 291-301, 1987.

[16] M. J. Forrestal and D. Y. Tzou, "A spherical cavity-expansion penetration model for concrete targets," International Journal of Solids and Structures, vol. 34, no. 31-32, pp. 4127-4146, 1997.

[17] Q. M. Li and X. W. Chen, "Dimensionless formulae for penetration depth of concrete target impacted by a non-deformable projectile," International Journal of Impact Engineering, vol. 28, no. 1, pp. 93-116, 2003.

[18] M.-Y. Wang, H.-J. Deng, and Q.-H. Qian, "Study on problems of near cavity of penetration and explosion in rock," Chinese Journal of Rock Mechanics and Engineering, vol. 24, no. 16, pp. 2859-2863, 2005.

[19] M.-Y. Wang, S.-L. Chen, and Y.-F. Pan, "Method of calculation for the penetration of a flat-nosed projectile in the rock (CONCRETE)," Acta Armamentarii, vol. 26, no. 1, pp. 46-52, 2005. 
[20] M.-Y. Wang, C.-Y. Shi, and S.-L. Chen, "Method of calculating critical spalling and penetration thickness of concrete slab of block under accident impact," Engineering Mechanics, vol. 26, no. 11, pp. 238-246, 2009.

[21] S. L. Chen, M. Y. Wang, and Y. F. Pan, "The method of calculation for penetration of a conical-nosed projectile in the rock(concrete) layers," Explosion and Shock Waves, vol. 24, pp. 7-15, 2004.

[22] M. Y. Wang, K. K. Tan, H. J. Wu, and Q. H. Qian, "New method of calculation of projectile penetration into rock," Chinese Journal of Rock Mechanics and Engineering, vol. 28, no. 9, pp. 1863-1869, 2009.

[23] M. Y. Wang, "Advances in the penetration of protective structures," Xiangshan-Science Conferences Report, 2013.

[24] T. Ge, M. Y. Wang, X. J. Li, and Y. F. Pan, "The penetration resistance and depth calculation for semi-infinite concrete target impacted by projectile," Shock and Vibration, vol. 27, pp. 107-110, 2008.

[25] P. F. Hadala, "Evaluation of empirical and analytical procedures used for predicting the rigid body motion of an earth penetrator," Tech. Rep. S-75-15, U.S. Army Waterways experiments station, Vicksburg, Miss, USA, 1975.

[26] A. L. Yarin, M. B. Rubin, and I. V. Roisman, "Penetration of a rigid projectile into an elastic-plastic target of finite thickness," International Journal of Impact Engineering, vol. 16, no. 5-6, pp. 801-831, 1995.

[27] Z. Wang, Analytical and numerical analysis of dynamic response of target penetration [Ph.D. thesis], Fudan University, Shanghai, China, 2005.

[28] M. J. Forrestal, B. S. Altman, J. D. Cargile, and S. J. Hanchak, "An empirical equation for penetration depth of ogive-nose projectiles into concrete targets," in Proceedings of the 6th International Symposium on Interaction of Nonnuclear Munitions with Structures, pp. 9-32, Panama City Beach, Fla, USA, May 1993.

[29] M. J. Forrestal, J. D. Cargile, and R. D. Tzou, "Penetration into concrete targets," in Advances in Numerical Simulation Technique for Penetration and Perforation of Solids, vol. 171, pp. 9-16, Applied Mechanics Division (AMD), American Society of Mechanical Enginees, 1993.

[30] H. J. Wu, Research on similar regularity of penetration mechanism and applied calculation [Ph.D. dissertation], PLA University of Science and Technology, Nanjing, China, 2008.

[31] Y. S. Tai, "Flat ended projectile penetrating ultra-high strength concrete plate target," Theoretical and Applied Fracture Mechanics, vol. 51, no. 2, pp. 117-128, 2009.

[32] M. J. Forrestal, "Penetration into dry porous rock," International Journal of Solids and Structures, vol. 22, no. 12, pp. 1485-1500, 1986.

[33] V. P. Alekseevskii, "Penetration of a rod into a target at high velocity," Combustion, Explosion, and Shock Waves, vol. 2, no. 2, pp. 63-66, 1969.

[34] F. F. Vitaman, N. A. Zlatin, and B. S. Loffe, "Deformable resistance of metals at velocities $10^{-6}-10^{2} \mathrm{~m} / \mathrm{s}$," Journal of Technical Physics, vol. 19, pp. 300-326, 1949.

[35] F. F. Vitman and N. A. Zlatin, "On collision of deformable bodies and its modeling: I. status and theory of the problem," Journal of Technical Physics, vol. 33, pp. 982-989, 1963.
[36] L. V. Belyakov, F. F. Vitman, and N. A. Zlatin, "On collision of deformable bodies and its modeling: II. The modeling of the impact of a sphere and a half-space," Journal of Technical Physics, vol. 33, pp. 990-995, 1963.

[37] L. I. Slepyan, "Calculation of the size of the crater formed by a high-speed impact," Soviet Mining Science, vol. 14, no. 5, pp. 465-471, 1978.

[38] H. Wang, H. W. Cai, Z. J. Wang, and R. C. Wang, "The concrete responses and resistance under high-velocity penetration," Journal of Projectiles, Rockets, Missiles and Guidance, vol. 4, pp. 54-59, 1997.

[39] Q. M. Tan, Dimensional Analysis, University of Science and Technology of China Press, 2005.

[40] C. Liu, Brittle material response to shock loading [Ph.D. dissertation], California Institute of Technology, Pasadena, Calif, USA, 1999.

[41] A. A. Kozhushko, I. I. Rykova, and A. B. Sinani, "Resistance of ceramics to penetration at impact velocity above $5 \mathrm{~km} / \mathrm{s}$," Journal de Physique IV, vol. 1, no. C3, pp. C3-117-C3-122, 1991.

[42] G. Hughes, "Hard missile impact on reinforced concrete," Nuclear Engineering and Design, vol. 77, no. 1, pp. 23-35, 1984.

[43] A. Haldar and H. A. Hamieh, "Local effect of solid missiles on concrete structures," Journal of Structural Engineering, vol. 110, no. 5, pp. 948-960, 1984.

[44] I. Kojima, "An experimental study on local behavior of reinforced concrete slabs to missile impact," Nuclear Engineering and Design, vol. 130, no. 2, pp. 121-132, 1991.

[45] Q. M. Li and X. W. Chen, "Penetration into concrete targets by a hard projectile," in Structures Under Shock and Impact VII, N. Jones, C. A. Brebbia, and A. M. Rajendran, Eds., WIT Press, Southampton, UK, 2002.

[46] D. E. Gault, "Displaced mass, depth, diameter, and effects of oblique trajectories for impact craters formed in dense crystalline rocks," The Moon, vol. 6, no. 1-2, pp. 32-44, 1973.

[47] S. B. Yu, G. C. Sun, and Q. M. Tan, "Experimental laws of cratering for hypervelocity impacts of spherical projectiles into thick targets," International Journal of Impact Engineering, no. 15, pp. 67-77, 1994. 

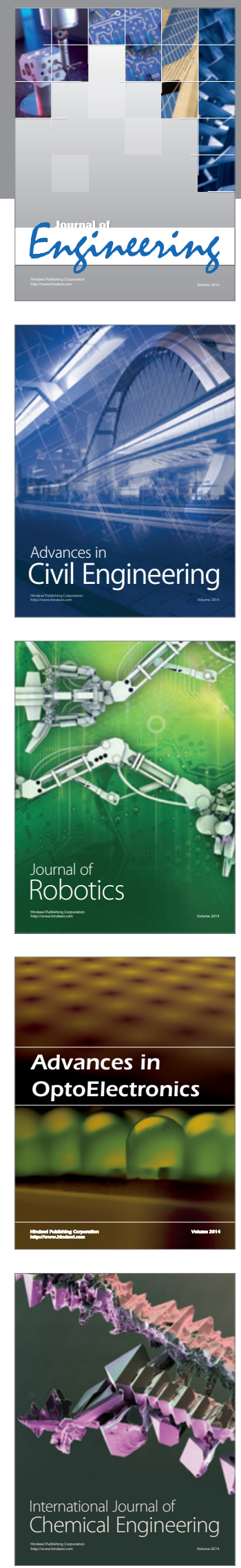

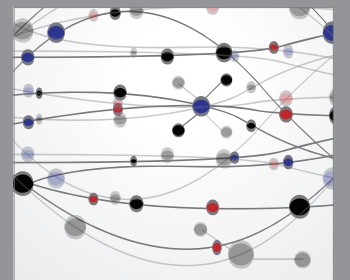

The Scientific World Journal
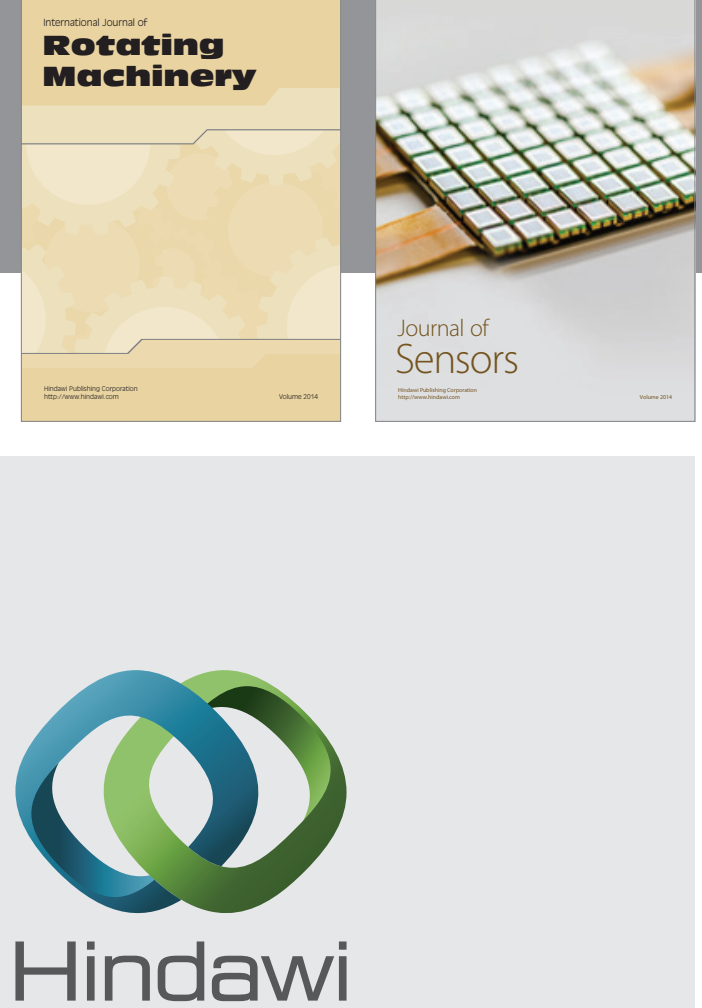

Submit your manuscripts at http://www.hindawi.com
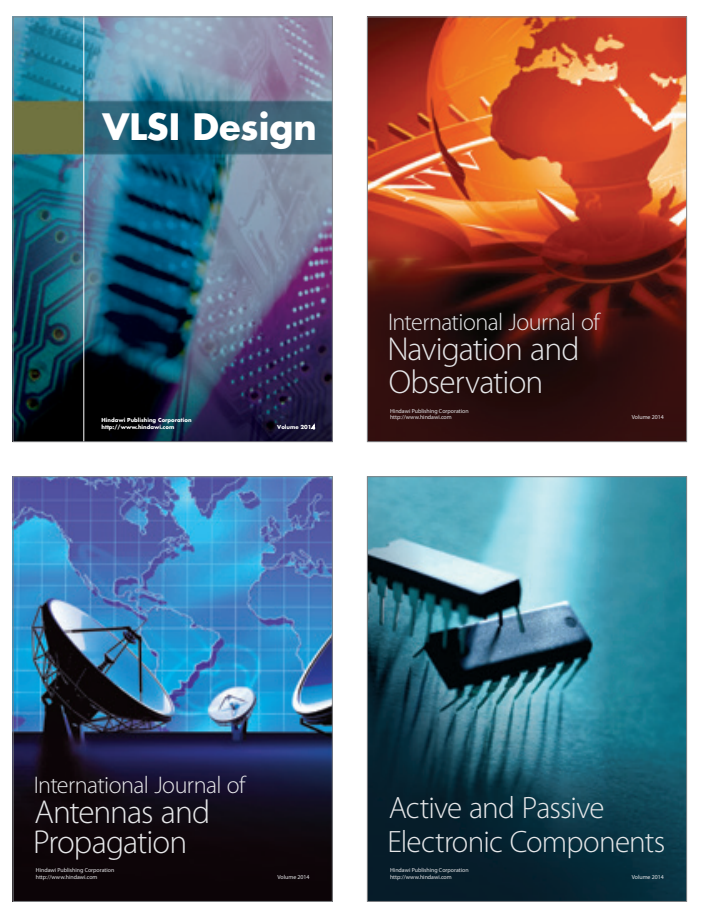
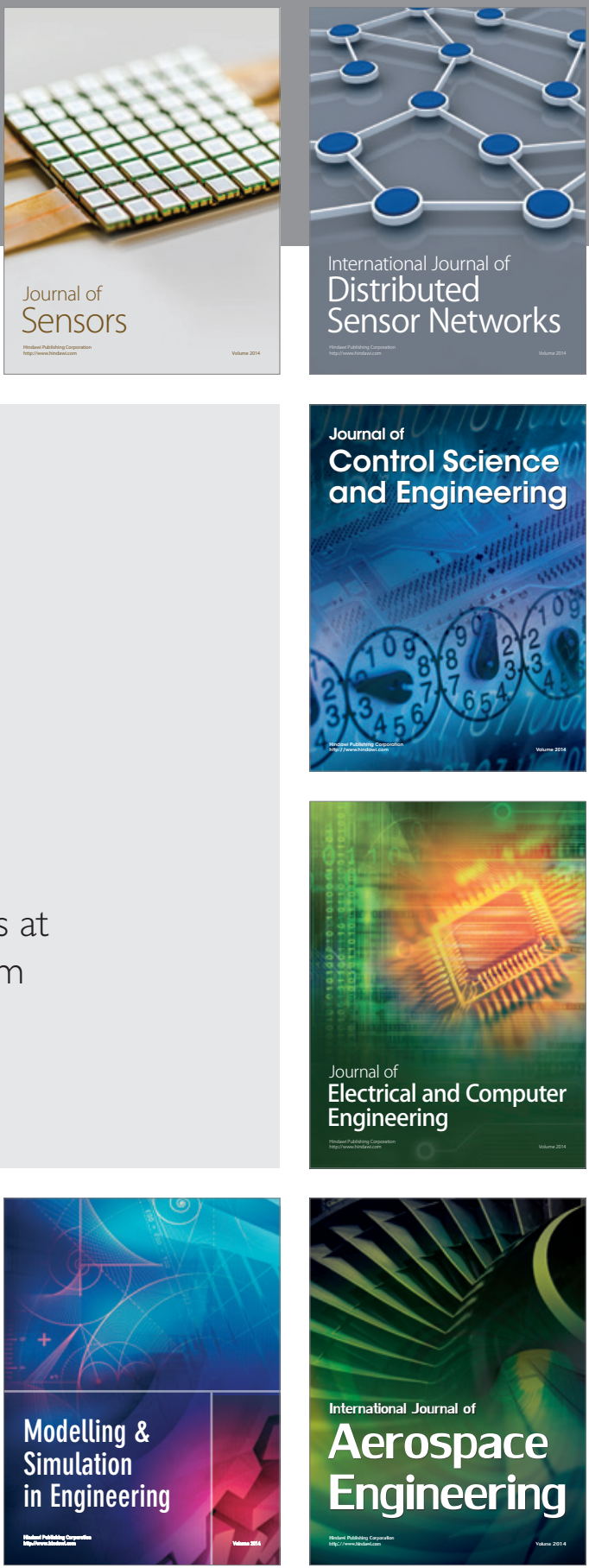

Journal of

Control Science

and Engineering
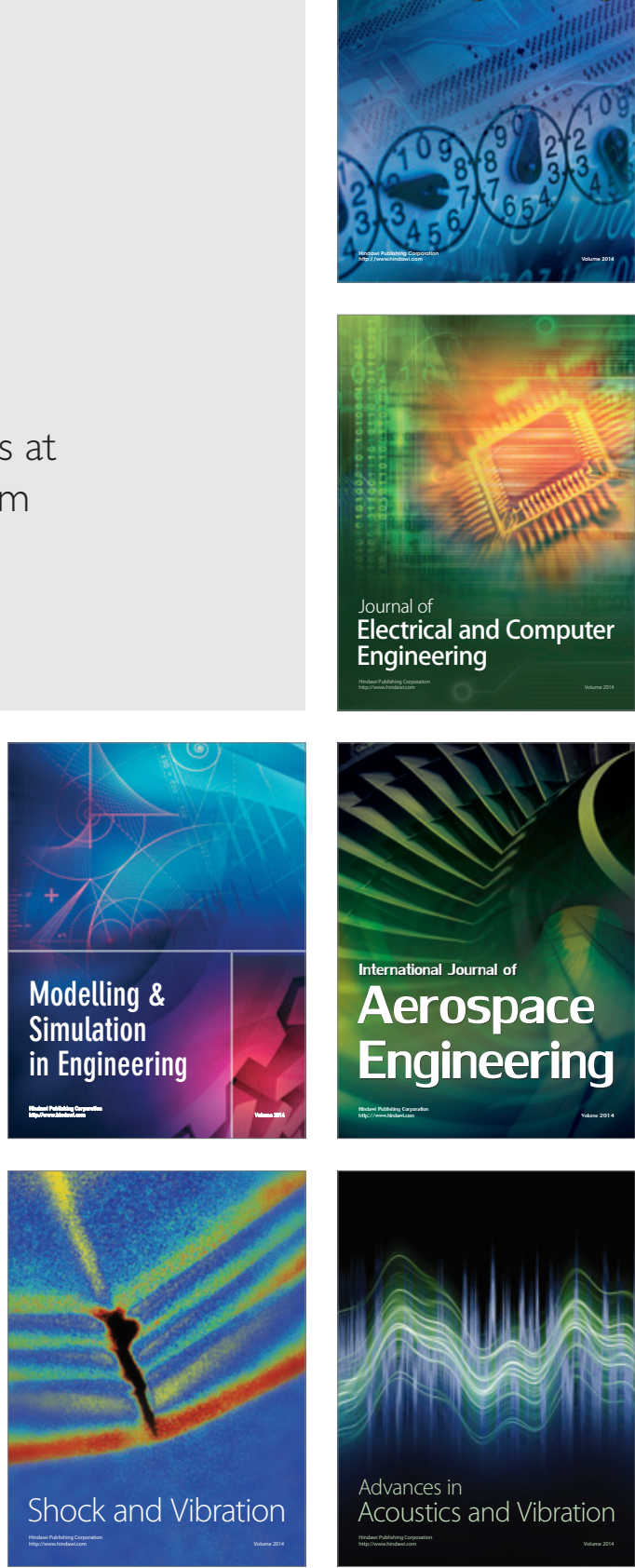